This is a postprint version of the following published document:

Cichocki, F., et al. Hybrid 3D model for the interaction of plasma thruster plumes with nearby objects, In: Plasma sources science and technology, 26, 125008, March 2019, 19 Pp.

DOI: https://doi.org/10.1088/1361-6595/aa986e

(C) 2017 IOP Publishing Ltd. 


\title{
Hybrid 3D model for the interaction of plasma thruster plumes with nearby objects
}

\author{
Filippo Cichocki, Adrián Domínguez, Mario Merino and Eduardo Ahedo \\ Equipo de Propulsión Espacial y Plasmas (EP2), ep2.uc3m.es, Universidad Carlos III de \\ Madrid, Leganés, Spain \\ E-mail: filippo.cichocki@uc3m.es
}

\begin{abstract}
This paper presents a hybrid PIC-fluid approach to model the interaction of a plasma plume with a spacecraft and/or any nearby object. Ions and neutrals are modeled with a particle-in-cell approach, while electrons are treated as a fluid. After a first iteration of the code, the domain is split into quasineutral and non-neutral regions, based on nonneutrality criteria, such as the relative charge density and the Debye length to cell size ratio. At the material boundaries of the former quasineutral region, a dedicated algorithm ensures that the Bohm condition is met. In the latter non-neutral regions, the electron density and the electric potential are obtained by solving the coupled electron momentum balance and Poisson equations. Boundary conditions for both the electric current and potential are finally obtained with a plasma sheath sub-code and an equivalent circuit model. The hybrid code is validated by applying it to a typical plasma plume-spacecraft interaction scenario, and the physics and capabilities of the model are finally discussed.
\end{abstract}

\section{Introduction}

As the use of electric thrusters onboard modern spacecraft continues to increase, understanding and predicting any integration issues early in the design process becomes essential. Electric thrusters, like the gridded ion thruster (GIT) or the Hall-effect thruster (HET) $[1,2]$, produce energetic plasma plumes that interact with the local electric and magnetic fields, and can induce electric charging, produce mechanical erosion/contamination and exert forces/torques on any object they interact with [3, 4]. While minimizing this interaction on different key components, such as the solar array and onboard sensors, is crucial in modern spacecraft designs, plasma plumes can also be used in exotic applications like in the ion beam shepherd (IBS) $[5,6,7,8]$, where a space debris object is gradually repositioned to a different orbit, through the ion push of an electric thruster plume, which is directed towards it by a "shepherd" spacecraft. The study of similar plasma jets is also relevant in other research fields, such as plasma material processing [9] and astrophysics [10, 11].

Plasma plumes from GITs and HETs are rarefied, with peak densities in the order of $10^{16}-10^{18} \mathrm{~m}^{-3}[1,2,12,13]$, and thus weakly collisional. In addition, they are characterized by very different dynamics for ions and electrons, with the latter being $4-5$ orders of magnitude lighter, and responding much more quickly to external perturbations. As a 


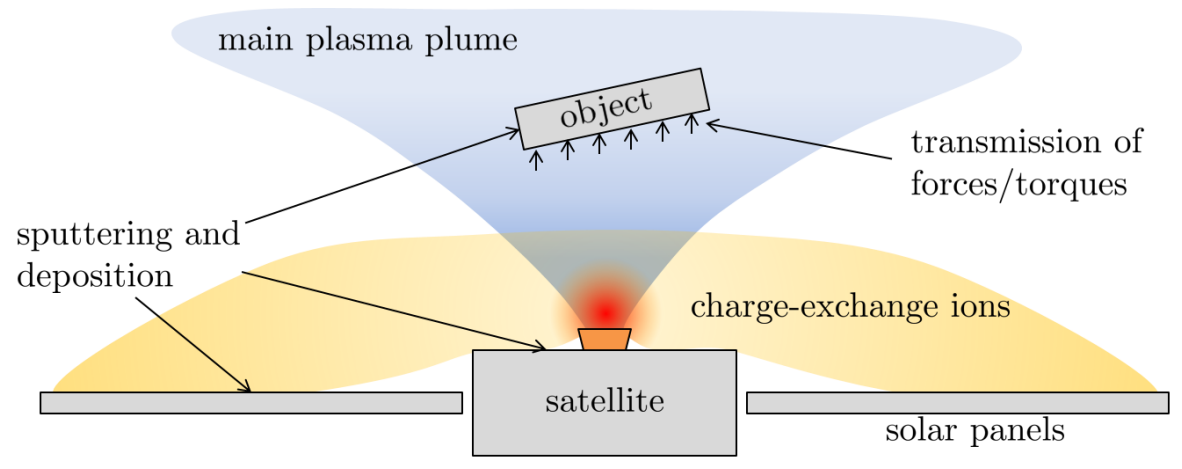

Figure 1: Schematic representation of the plasma plume interaction with the emitting $\mathrm{S} / \mathrm{C}$ and with an object immersed in it

consequence, mass and momentum are mostly due to ions, while the self-consistent electric fields depend mostly on the electrons dynamics, and are strongly affected by the presence of externally applied magnetic fields, so that analyses must distinguish between magnetized and unmagnetized plasma plumes. In general, a plasma plume features a 'near region' (extending up to a few thruster radii from its exit surface) and a 'far-region' $[12,13]$. In the former, the plasma is markedly non-homogeneous, with non-negligible 3D effects due to (I) particle collisions, such as the charge-exchange (CEX) ones [14, 15] between ions and neutrals, (II) plume quasineutralization, and (III) the applied electric and magnetic fields. In the farregion, on the other hand, a smooth, more rarefied, single-peaked plume profile generally forms, and the above effects become negligible with respect to the plume kinetic energy. The subsequent expansion of the plasma is here mainly governed by the residual thermal pressure, and the self-consistent ambipolar electric field. This paper focuses on unmagnetized plumes and presents a model covering both the plume near and far regions.

The above described plume physics can be modeled following different approaches, going from multi-fluid models to fully kinetic ones. Table 1 lists relevant plume models and codes. Multi-fluid models operate directly with the relevant macroscopic variables and feature at least one fluid per existing species. The main issue they present comes from the weak plasma collisionality and the consequent lack of thermodynamic equilibrium. Deviations from the Maxwellian distribution function may recommend modeling a given species as two or more fluids (distinguishing for instance between slow and fast ions). More importantly perhaps, fluid approaches raise uncertainties on the closure of the fluid equations and the correct expression for the pressure tensors, the heat flux vectors, and the resistive forces. The problem is usually more relevant for electrons, since often ion pressure is negligible compared to electron pressure. The simplest fluid models close electron fluid equations at the level of the momentum equation postulating an isothermal or polytropic equation of state (e.g. a generalized Boltzmann relation). Examples are the collisionless, two-fluid models of Refs. [12], [13], [16], [17] and [18], which approximate well the plume far-region, as shown by comparison with both experiments [16, 17, 19] and more complex kinetic simulations [20]. A better suited multi-fluid model covering the near-region is that of Ref. [21], which includes collisional effects, several fluids per ion species, and a Fourier law for the heat flux of the 
magnetized electrons.

At the other end of the modeling approaches are the fully kinetic models [22], in which the velocity distribution functions are obtained by solving Boltzmann equation in a 6-dimensional phase-space (position and velocity). In general these models are totally unaffordable, except for very simplified configurations. Full particle-in-cell (PIC) models $[23,24]$ can be considered as a very coarse approximation to fully kinetic models. They follow an alternative Lagrangian-Eulerian approach, in which electron and ion populations are modeled as sets of macro-particles subject to the action of electric and magnetic fields and occasional collisions. Macroscopic magnitudes are computed at the nodes of dedicated meshes extending along the physical domain. Full-PIC models present the drawback of a very small time step and cell size, as dictated by the electron small inertia and fast dynamics. Even with good parallelization, they require typically weeks or months to reproduce faithfully ion dynamics in a plasma thruster. The computational cost is even more severe for thruster plumes and their interaction with the spacecraft, which is an inherently three-dimensional problem, featuring plasma density variations of several orders of magnitude, and requiring simulation domains of a few meters in all directions. Moreover, the statistical PIC noise tends to grow as the plasma expands and the number of macro-particles per cell diminishes, and this growth can be mitigated only with the use of complex population control algorithms.

Table 1: Existing plasma plume codes and their capabilities.

\begin{tabular}{||c|c|c|c|c|c|c||}
\hline $\begin{array}{c}\text { Code name } \\
\text { or author }\end{array}$ & References & $\begin{array}{c}\text { Publication } \\
\text { date }\end{array}$ & $\begin{array}{c}\text { Model } \\
\text { type }\end{array}$ & $\begin{array}{c}\text { Structured } \\
\text { mesh }\end{array}$ & $\begin{array}{c}\text { Non } \\
\text { neutral }\end{array}$ & $\begin{array}{c}\text { Electron } \\
\text { fluid closure }\end{array}$ \\
\hline \hline Parks & {$[16]$} & 1979 & 2D fluid & yes & no & Boltzmann \\
\hline Korsun & {$[18,25]$} & 1997 & 2D fluid & yes & no & Boltzmann \\
\hline Oh & {$[26]$} & 1999 & 3D hybrid & yes & no & Boltzmann \\
\hline Ashkenazy & {$[17]$} & 2001 & 2D fluid & yes & no & Boltzmann \\
\hline SPIS & {$[27,28,29]$} & 2001 & 3D hybrid & no & yes & Boltzmann \\
\hline CNES & {$[30]$} & 2002 & 2D hybrid & yes & yes & Boltzmann \\
\hline AQUILA & {$[31,32]$} & 2003 & 3D hybrid & no & no & polytropic \\
\hline DRACO & {$[33]$} & 2004 & 3D full-pic & yes & yes & n/a \\
\hline Taccogna & {$[34]$} & 2011 & 3D hybrid & yes & no & polytropic \\
\hline USC & {$[23,24]$} & 2014 & 3D full-pic & yes & yes & n/a \\
\hline SUGAR & {$[35,36]$} & 2014 & 3D hybrid & no & no & Boltzmann \\
\hline EASYPLUME & {$[12,13]$} & 2015 & 2D fluid & yes & no & polytropic \\
\hline $\begin{array}{c}\text { Greifswald } \\
\text { university }\end{array}$ & {$[37]$} & 2014 & 3D full-pic & yes & yes & n/a \\
\hline Hall2De & {$[38]$} & 2015 & 2D hybrid & yes & yes & polytropic \\
\hline $\begin{array}{c}\text { New Mexico } \\
\text { State university }\end{array}$ & {$[39]$} & 2015 & 3D hybrid & yes & yes & heat flux \\
\hline SM/SMURF & {$[40]$} & 2016 & 3D hybrid & yes & yes & polytropic \\
\hline
\end{tabular}

An intermediate approach between full-PIC and multi-fluid models, are the hybrid models $[27,28,29,31,34,35,38,39,40,41,42$, 43], in which heavy particles are PIC- 
modeled, while electrons are treated as one or more fluids. This last choice allows to overcome the severe constraints dictated by the electron dynamics, and to increase the computational time step by 3-4 orders of magnitude. Furthermore, while full-PIC codes are inherently nonneutral electrically and must solve the electric field from the Poisson equation, hybrid codes can assume quasineutrality in part of or in the whole simulation domain, thus allowing larger cell sizes and further reducing the computational time. Of course, hybrid codes present the drawback of a limited accuracy in simulating the electron dynamics (the most common fluid closure being the isothermal or polytropic electrons) but, at present and at least for most research purposes, they constitute the best compromise in terms of accuracy, complexity and computational cost for plasma thruster and plume simulations.

This paper presents a flexible 3D hybrid PIC/fluid model and code of the interaction of a rarefied plasma plume with the spacecraft and/or any nearby object. A preliminary version of the code, named EP2PLUS ('Extensible Parallel Plasma Plume Simulator'), has already been presented in Ref. [42]. The main features are the following. First, multiple macroparticle populations are considered in order to address not only the particle mass and charge, but also different ranges of energy, in a computationally optimal manner. Second, both Direct Simulation Monte Carlo (DSMC) and deterministic schemes are implemented, depending on the type of collision to be modeled. Third, population control algorithms are implemented in each cell in order to improve the macroparticles number and weight and thus reduce the statistical noise inherent to PIC formulations [44, 45, 46]. Fourth, while standard volumetric weighting is used in inner cells, more accurate surface weighting is implemented at the material boundary cells [47, 48]. Fifth, a dynamic splitting algorithm for quasineutral and non neutral regions is applied to compute the electric field in the highly inhomogeneous density regions of the domain. Sixth, Bohm condition forcing algorithms are implemented in quasineutral material boundaries, in order to compute correctly the ion fluxes to the walls $[47,48]$. Seventh, an equivalent electric circuit is used to deal efficiently with the different conductive walls of the spacecraft and the thruster. And eighth, a weakly-collisional electron fluid model is proposed, which overcomes the limitations of the typical Boltzmann relation while simultaneously yielding the electron currents, and therefore allowing the simulation of the current neutralization at the thruster exit. Finally, the presented model is validated by applying it to a typical spacecraft-plume interaction scenario, and its physics and capabilities are discussed.

Regarding the paper structure, Sec. 2 presents the features of the PIC model, while Sec. 3 describes the electron fluid model. The quasineutral fluid closure is presented in Sec. 4, while the approach followed for solving non-neutral plasma regions is reported in Sec. 5 . Sec. 6 summarizes the model, and, finally, Sec. 7 presents and discusses the results of the spacecraftplume interaction. 


\section{The PIC model for heavy species}

In the PIC model, the distribution functions of the heavy species are discretized in both position and velocity, following a Lagrangian approach with Dirac delta functions [49]:

$$
F(\boldsymbol{r}, \boldsymbol{v}, t) \approx \sum_{p=1}^{N} \delta\left(\boldsymbol{r}-\boldsymbol{r}_{p}(t)\right) \delta\left(\boldsymbol{v}-\boldsymbol{v}_{p}(t)\right)
$$

where $\boldsymbol{r}_{p}$ and $\boldsymbol{v}_{p}$ are the position and velocity vectors of the $p^{\text {th }}$ macro-particle. In order to reduce the computational cost of the simulation, at the cost of a higher statistical noise, a limited number $N$ of macro-particles is used, each of them representing a very large number $W$ of elementary particles, referred to as the macro-particle weight. The motion of each macro-particle in the electromagnetic field is solved by integrating the corresponding Newton equation, while its collisions with other macro-particles and with the material surfaces of the domain are simulated as instantaneous events (the real interaction time is indeed much shorter than the integration time step). The distribution functions are then weighted to the nodes of a dedicated PIC mesh in order to obtain Eulerian weighted properties at specific positions, used by the fluid closure model to obtain the self-consistent fields. This hybrid Lagrangian-Eulerian approach permits to reduce to $O(N)$ the number of elementary operations to be carried out every time step, versus the $O\left(N^{2}\right)$ number of operations of molecular dynamics codes [49].

\subsection{General features}

As shown in Table 1, hybrid codes can make use of either unstructured or structured meshes. In this work, a structured mesh is used, given its higher computational efficiency in terms of macro-particles sorting algorithms, at the cost of a lower flexibility when dealing with complex object geometries. In a structured mesh, each position vector is identified by a set of 3 computational coordinates $(\xi, \eta, \zeta)$, which have a bijective relation with the corresponding physical coordinates $(x, y, z)$, as shown in Fig. 2. If $N_{\xi}, N_{\eta}, N_{\zeta}$ are the numbers of nodes along the three coordinate directions, then the computational coordinates vary respectively in the ranges $\left[0, N_{\xi}-1\right],\left[0, N_{\eta}-1\right]$ and $\left[0, N_{\zeta}-1\right]$. A typical cell features a side of approx. $1 \mathrm{~cm}$, whereas the Debye length varies from fractions of $\mathrm{mm}$, to tens of $\mathrm{cm}$. Regarding the number of nodes, this is around $10^{6}$, i.e. the simulation domain typically feature 100 nodes along each coordinate direction.

In order to maintain the stability of the integration scheme, the PIC time step $\Delta t$ is selected so that the fastest macroparticles do not cross more than one cell per time step [50]. The particle mover is based on Boris' CYLRAD algorithm [51], a generalization of the second order leap frog integration, and hence with velocity and position of the macro-particles referring to interleaved time points (separated by $\Delta t / 2$ ). In the following, $k$ represents the current PIC time step, so that macroparticles positions are known at time $k$, while velocities at time $k-1 / 2$.

Macro-particles are grouped into $L$ different populations, each of them stored in a dedicated computational list, in terms of their atomic mass $m$, their charge number $Z$, and 


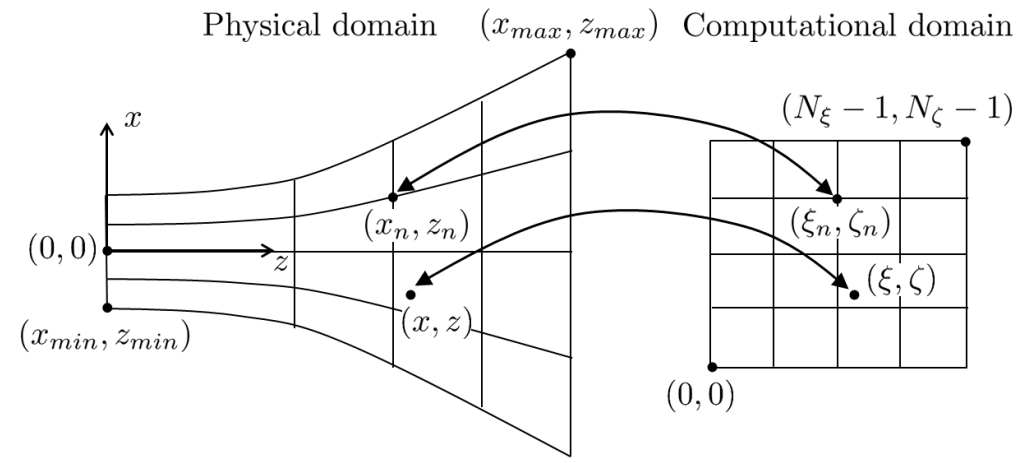

(a)

Figure 2: Physical and computational domains, associated to the PIC mesh. For the sake of clarity, a 2-dimensional $x, z$ plane slice of the domain is shown

their origin or characteristic kinetic energy. The division of macro-particles of the same type and charge in terms of this last property is used to achieve better statistics and can also enable the use of different $\Delta t$ for fast and slow particles as a means to accelerate the simulation. The clearest example is that of the CEX collision, in which the 'slow ions' and the 'fast neutrals' produced by this event are stored into dedicated populations, independent of the 'fast ions' and 'slow neutrals' ones.

Once the macro-particles of each population have been moved, they are assigned or sorted to the corresponding cell nodes. In a structured mesh, the corresponding cell indices are efficiently obtained as the integer part of the macro-particles computational coordinates. A first order cloud-in-cell (CIC) shape [52] is then considered to weight the macro-particle to each mesh node. The particle density of a generic population is then obtained as:

$$
n=\frac{1}{\Delta V} \sum_{p=1}^{N} W_{p}\left(1-\left|\xi_{p}^{\prime}\right|\right)\left(1-\left|\eta_{p}^{\prime}\right|\right)\left(1-\left|\zeta_{p}^{\prime}\right|\right)
$$

where $\Delta V$ is the volume associated to the PIC mesh node (which for inner nodes of a Cartesian mesh coincides with the physical cell volume), $N$ is the number of macro-particles of the considered population belonging to adjacent cells, and $\left(\xi_{p}^{\prime}, \eta_{p}^{\prime}, \zeta_{p}^{\prime}\right)$ are the computational coordinates of the $p^{\text {th }}$ macro-particle, relative to the considered node.

\subsection{Macro-particles collisions}

Although the plasma plume is only weakly collisional, it is still affected by a large variety of collisional processes, especially in the near-region. For our intended applications the most relevant ones are:

- Ionization collisions: $A+e \rightarrow A^{+}+2 e, A+e \rightarrow A^{++}+3 e$, and $A^{+}+e \rightarrow A^{++}+2 e$.

- Symmetric and pure CEX collisions: $A^{+}$(fast) $+A$ (slow) $\rightarrow A^{+}$(slow) $+A$ (fast) and $A^{++}($fast $)+A($ slow $) \rightarrow A^{++}($slow $)+A($ fast $)$.

Regarding the former, higher ionization degrees are irrelevant in most plasma plumes, given their increasing ionization energies and hence decreasing reaction rates. For what 
concerns the CEX collisions, the considered ones are the resonant-symmetric reactions, with no momentum exchange. Such reactions are the dominant collisions in a plasma plume and have the highest cross sections, as shown in Refs. [13], [14], [15], and [53]. The assumption of a zero momentum exchange is a good approximation for resonant CEX, also accepted in more complex ion-neutral collision models, like that of Ref. [53], in which the collision outcome is either a pure CEX or an elastic momentum exchange collision (MEX).

MEX collisions have generally little importance in a plasma plume. Ion-ion MEX collisions, due to Coulomb interaction, modify the affected particles relative velocity (for an ion species emitted by a plasma thruster, this amounts to fractions of $\mathrm{eV}$ in terms of energy), which is much smaller than their absolute velocity (thousands of $\mathrm{eV}$ ), so that their effect is clearly negligible. MEX collisions between ions and neutrals, or between ions of different charge, on the other hand, can have larger effects. However, their importance with respect to that of the CEX is still small, as shown in Ref. [54], especially when it comes to determine the backscattering ion flux towards the satellite, almost entirely constituted by slow CEX ions.

Although excitation collisions are an important factor of energy loss inside a plasma thruster, their effect in the dynamics of a plasma plume is also negligible. From a PIC point of view, these collisions create a macro-particle belonging to a different excited population, with possibly different collision cross sections, but with the same charge number $Z$. This is clearly a second order effect, since only the collision properties (but not the trajectory) of those few macro-particles that suffer an excitation collision are affected. Finally, recombination collisions are also neglected, given their low frequency in a cold rarefied plasma plume.

In the following, the cell-wise algorithms for the considered collisions are presented.

2.2.1. Ionization collisions. The approach considered here is similar to that of HP-HALL [44], and takes into account the different weights of the neutral and ion populations. In order to describe the general approach, we illustrate the particular case of $A+e \rightarrow A^{+}+2 e$. First, all neutral macro-particles (i.e. the input population) are sampled and their weight reduced due to ionization (deterministic sampling). Then, the ionization rate $R_{01}\left(T_{e}\right)$ is evaluated at the cell center with the Drawin model [55], with the knowledge of the electron temperature $T_{e}$ (the Drawin model and the Bell model [56] are used respectively for the other reactions $A+e \rightarrow A^{++}+2 e$ and $A^{+}+e \rightarrow A^{++}+3 e$ ).

The mass of new singly-charged ions, $\Delta m_{i}$, to be generated in the cell of physical volume $V_{c}$ during the time $\Delta t$ is

$$
\Delta m_{i}=n_{e} n_{n} m R_{01}\left(T_{e}\right) V_{c} \Delta t
$$

where $n_{e}$ and $n_{n}$ are the electron and neutral densities at the cell center. Then, the average number of new ion macro-particles is $\Delta N=\Delta m_{i} /\left(m W_{g e n}\right)$, rounded appropriately, where $W_{\text {gen }}$ is the generation weight associated to both the cell and the output ion population (refer to Sec. 2.5). The position of the new ion macro-particles is uniformly distributed within the cell and their velocity is sampled from a Maxwellian distribution, with mean velocity and temperature equal to those of the neutrals. Finally, the weights of all the neutral macroparticles in the cell are updated by reducing them proportionally to their values, with a total reduction in the cell of $-\Delta m_{i} / m$. 
2.2.2. CEX collisions. Given the two input populations (e.g. fast ions and slow neutrals), the first step is to sample the macro-particles that undergo a CEX collision. A Direct Simulation Monte Carlo (DSMC) approach has been implemented: existing macro-particle pairs are checked for collision (each pair constituted by one ion and one neutral macro-particle), and a specific pair collides if a random number $U$ is lower than its collision probability $p_{c, p a i r}$ :

$$
p_{c, p a i r}=\frac{1-\exp \left(-\sigma\left(v_{r}\right) n_{n} v_{r} \Delta t\right)}{N_{n}}
$$

where $\sigma$ is the collision cross section, $v_{r}$ is the relative velocity between the macro-particles of the pair, $n_{n}$ is the elementary number density of the neutral population, $N_{n}$ the number of neutral macro-particles inside the cell, and $\Delta t$ must not be larger than the expected collision time. Given the high number of pairs to be checked $\left(N_{i} N_{n}\right)$, a more efficient DSMC version [49] has been adopted that limits this number to $p_{c, \text { max }} N_{i} N_{n}$, with $p_{c, \text { max }}=p_{c, \text { pair }}\left(\left|v_{r} \sigma\left(v_{r}\right)\right|_{\text {max }}\right)$ representing the maximum expected collision probability for the pair. The random number $U$ is then compared to the ratio $p_{c, \text { pair }} / p_{c, \text { max }}$, thus resulting into a more efficient macro-particles sampling (e.g. less probability checks, with a higher collision acceptance probability).

The functional dependence of the CEX cross section $\sigma\left(v_{r}\right)$ can be found in Appendix A. After the sampling step, new macro-particles have to be generated in the slow ion and fast neutral populations. Referring to Fig. 3, these are uniformly distributed within the cell and have the generation weight of the corresponding cell and output population.

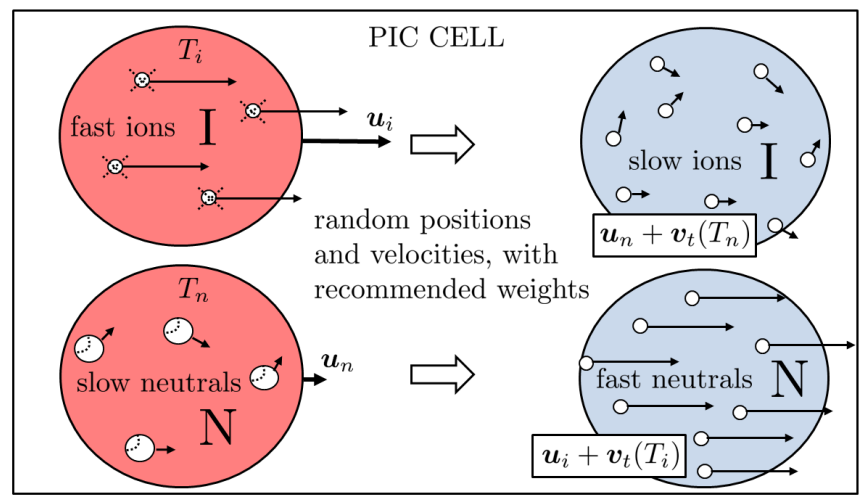

Figure 3: CEX DSMC sampling (left) and generation (right). The sampled ion macro-particles are removed from the simulation, and the weights of the sampled neutrals are reduced by the corresponding amount.

Regarding their velocities, these are sampled from a local Maxwellian distribution with mean velocity and temperature given by the corresponding input population. This means that the slow ions feature a fluid velocity and temperature equal to that of the slow neutrals, while the fast neutrals have the same fluid properties of the fast ions.

Finally the input populations are updated pair by pair, meaning that the weight of the heavier macro-particle is updated by subtracting the weight of the lighter macro-particle, which is removed from the simulation. An acceptance-rejection scheme prevents the algorithm from producing very small residual macro-particles when the input weights are very similar, while preserving mass on average. 


\subsection{The surface interaction and particle injection}

Macro-particles can interact with boundary faces in boundary cells, representing material, injection or sink surfaces. The use of a structured mesh allows to identify any cellface with a set of 3 indices, which are used to fill a 3-D matrix containing the corresponding surface types, each one identified by a specific integer value. Table 2 summarizes the surface types considered here.

\begin{tabular}{|c|c|c|}
\hline Element type & Effects on ions & Effects on neutrals \\
\hline \hline Transparent & none & none \\
\hline Particle sink & removal & removal \\
\hline Injection & stochastic injection & stochastic injection \\
\hline Material wall & recombination & reflection \\
\hline
\end{tabular}

Table 2: Existing surface types. Macro-particles are injected according to given injection profiles from the injection cells.

By monitoring the variations of the integer part of a macro-particle computational coordinates, a crossing-detection algorithm verifies if it has crossed a boundary face. If this happens, the macro-particle is sorted for surface interaction, consisting in applying on it the effect of surface interaction, and adding its contribution to surface-weigthing in that face.

2.3.1. Effects on macro-particles. They depend on the surface type. First, if the macroparticle crosses a sink boundary-face, such as a vacuum boundary, the macro-particle is simply removed from the domain.

Second, at injection boundary faces, like the exit surface of a plasma thruster, macroparticles are stochastically generated with the algorithms of Ref. [49]. These consider an injected particle flux $g_{i n j}$ and a probability distribution function for injection

$$
f_{i n j}(\boldsymbol{v}) \propto v_{\perp} \exp \left(-\frac{m\left|\boldsymbol{v}-\boldsymbol{u}_{i n j}\right|^{2}}{2 T_{i n j}}\right)
$$

where $\boldsymbol{u}_{i n j}$ and $T_{i n j}$ are the injection fluid velocity and temperature, and $v_{\perp}$ is the cell-face perpendicular component of the macro-particle velocity. The injected macro-particle position is uniformly distributed across the injection face, and the number of injected particles depend on $g_{i n j}$ and on the generation weight $W_{\text {gen }}$ of the injection cell. In order to simulate a continuous injection, each injected macro-particle is advanced, along its velocity direction, a random fraction of the PIC time step. For what concerns macro-particles (ions or neutrals) crossing an injection face from within the plasma, they are simply re-injected as additional neutrals by the above described algorithm.

When crossing a material boundary face, ion macro-particles recombine into neutrals, while neutral macro-particles suffer either a specular or a diffuse reflection, with a defined probability. While the neutral specular reflection is simply simulated by inverting the normal velocity component of the macro-particle, the neutral re-injection due to both ion 
recombination or neutral diffuse reflection, is carried out independently for each impacting population. The mean re-injection energy is

$$
\bar{E}_{\text {reinj }}=\alpha_{W} 2 T_{W}+\left(1-\alpha_{W}\right) \bar{E}_{i m p}
$$

where $T_{W}$ is the wall temperature (in energy units), $\alpha_{W}$ is a wall accommodation coefficient, and $\bar{E}_{i m p}$ is the time-averaged wall-impact kinetic energy (per elementary particle) for the impacting population.

Regarding the angular distribution of the emission, a thermal cosine emission law is assumed for the injection probability distribution function, which is equivalent to using Eq. 5, with $\boldsymbol{u}_{i n j}=\mathbf{0}$, and $2 T_{i n j}=\bar{E}_{\text {reinj }}$. Just like for injection and collisions, the re-injected neutral macro-particles feature the generation weight $W_{\text {gen }}$ of the corresponding cell.

For those material interfaces immersed in a quasineutral region (see Sec. 5), the sheath potential fall must be taken into account in order to determine $\bar{E}_{\text {imp }}$ (Eq. 6) and the energy flux to the wall of ion macroparticles. If $\phi_{S}$ is the sheath edge potential, $\phi_{W}$ is the wall potential, and $E_{\perp S}$ and $E_{\| S}$ the kinetic energies (per elementary particle) normal and parallel to the sheath edge, ion macroparticles reach the wall if

$$
E_{\perp W}=E_{\perp S}+e Z\left(\phi_{S}-\phi_{W}\right)>0
$$

and with an impact energy (per elementary particle) $E_{i m p}=E_{\perp W}+E_{\| S}$. Otherwise they are treated as specularly reflected from the sheath edge.

2.3.2. Surface-weighting. Extended Surface Weigthing algorithms were introduced in Refs. [48] and [47] and provide more accurate results for macroscopic variables than volumetric weighting at boundary faces and nodes. Simple surface weighted density and particle flux vector are defined as:

$$
\begin{aligned}
n_{s w} & =\frac{1}{\Delta t \Delta S} \sum_{p=1}^{N_{h i t}} \frac{W_{p}}{\left|v_{\perp, p}\right|} \\
\boldsymbol{g}_{s w}=(n \boldsymbol{u})_{s w} & =\frac{1}{\Delta t \Delta S} \sum_{p=1}^{N_{h i t}} \frac{W_{p} \boldsymbol{v}_{p}}{\left|v_{\perp, p}\right|}
\end{aligned}
$$

where $v_{\perp, p}$ is the $p^{\text {th }}$ macro-particle perpendicular velocity (with respect to the cell-face), $N_{\text {hit }}$ is the number of hitting (from the plasma) or emitted (towards the plasma) macro-particles in the current time step, and $\Delta S$ is the boundary face area. Since few particles typically cross a boundary face in a single time step, the extended time-averaged version

$$
\bar{n}_{s w}^{(k)}=\frac{\left(\Delta k_{a v g}-1\right) \bar{n}_{s w}^{(k-1)}+n_{s w}}{\Delta k_{a v g}}
$$

is used for the density, and similarly for other variables, with $\Delta k_{a v g}$ the averaging number of time steps, and $k$ and $k-1$ meaning two consecutive time steps. These time-averaged surfaceweighted ion and plasma properties are finally interpolated from cell-faces to mesh nodes at the material boundaries. 


\subsection{The Bohm condition forcing}

In most or all quasineutral material boundary faces (refer to Sec. 5) there is a 'negative' sheath with $\phi_{W}<\phi_{S}$, thus attracting ions. A stable stationary sheath requires ions to fulfill the kinetic Bohm condition [57, 48, 47], at the sheath edge

$$
\sum_{s=1}^{L} \int_{0}^{\infty}\left(\frac{Z_{s}}{T_{e}}-\frac{Z_{s}^{2}}{m_{s} v_{\perp}^{2}}\right) F_{s}\left(v_{\perp}\right) d v_{\perp} \geq 0,
$$

where $L$ is the total number of particle populations and $F_{s}$ is the distribution function of the $s^{\text {th }}$ population at the sheath edge (integrated over parallel velocities). The surface-weighted PIC version of the Bohm condition is

$$
P \equiv \frac{1}{\Delta t \Delta S} \sum_{s=1}^{L} \sum_{p=1}^{N_{h i t}} \frac{W_{p}}{\left|v_{\perp, p}\right|}\left(\frac{Z_{s}}{T_{e}}-\frac{Z_{s}^{2}}{m_{s} v_{\perp, p}^{2}}\right)>0,
$$

where $m_{s}$ is the elementary mass of the $s^{\text {th }}$ population, and $\delta \phi$ is the electric potential drop applied in a virtual pre-sheath region (not simulated by the PIC).

It is also well known that the electric field perpendicular to the wall changes quickly near the sheath edge. Ahedo and Parra [47] showed that the Bohm condition was far from being satisfied for typical cell sizes in a quasineutral PIC code and proposed the Bohm condition forcing (BCF) algorithm [48, 47] as the most efficient way to fulfill Eq. 10. The algorithm can be understood as a virtual transition layer between the boundary simulation domain and the sheath (much thinner than the cell size and much thicker than the Debye length) that adapts the electric potential to satisfy the Bohm condition by acting on $n_{e}$. Whenever $P<0$ in Eq. 11 , the algorithm introduces a potential fall $\delta \phi$, which increases the perpendicular velocity $v_{\perp, p}$ in order to have $P \simeq 0$. Ahedo et al.[47] showed that the correction delta-phi required to satisfy the Bohm condition decreases over time.

\subsection{The PIC population control}

In PIC codes, controlling the macro-particle number and weight is fundamental to have low statistical noise, accurate collision algorithms and optimal computational cost. The goals of population control algorithms are therefore to (I) ensure that the number of macro-particles per cell be within a desired interval $\left[N_{\min }, N_{\max }\right]$, and (II) to minimize the weight dispersion within each cell. Possible actions to achieve them are:

- Use of a non-uniform physical mesh adapted to the local species density. Larger cells improve velocity-space resolution, at the cost of a lower physical-space resolution.

- Active control of generated macro-particle number or weight in collision and surface interaction algorithms. This approach is conditioned to the existence of sufficient collisional and surface interaction events in or near the PIC cells where the population must be controlled.

- Use of a weight re-normalization algorithm, which either splits or groups existing macroparticles, while conserving both their overall momentum and energy [58, 59]. 
At present, the first two solutions have been implemented. An example of the first one was illustrated in Ref. [42] where a conical mesh reduced dramatically the numerical noise downstream of a plasma plume. The second approach is described here.

Secs. 2.2 and 2.3, already mentioned the use of a generation weight $W_{\text {gen }}=W_{\text {gen }}(\boldsymbol{r})$, for each cell and particle population. A dedicated algorithm updates such a generation weight in all cells, by monitoring the current number of macro-particles per cell $N_{c}$, and the average weight of the existing macro-particles $\bar{W}$ in the cell. Let $N_{t g}$ be a targeted number of macroparticles per cell, within the interval $\left[N_{\min }, N_{\max }\right]$. Then, if at least one macro-particle has been generated or injected in the considered cell in the latest time step, the generation weight is simply updated as $W_{g e n}=\bar{W} \bar{N}_{c} / N_{t g}$. Thus the generation weight represents the target value that a renormalization algorithm should consider, in splitting or merging macro-particles, in order to achieve the targeted number of macroparticles per cell. The use of the average number of macro-particles in the cell permits reducing the oscillations in the generation weight, thus resulting into a more reliable and robust algorithm.

\section{The electron fluid model}

The fluid model for electrons, complemented with Poisson's equation for the electric potential $\phi$, permits computing their density $n_{e}$, temperature $T_{e}$, and current density $j_{e}=$ $-e n_{e} \boldsymbol{u}_{e}$. As commented in the Introduction, since electrons are weakly-collisional, local thermodynamic equilibrium cannot be invoked and the closure of the fluid equations is delicate. If collisions are introduced through standard resistive terms, the main concern is in the expressions of the pressure tensor $\mathcal{P}_{e}$ (in the momentum equation) and of the heat flux (in the energy equation), in the collisionless limit. Both should be derived from a kinetic approach, and several attempts are under progress in this regard, both for magnetized and unmagnetized plumes [23, 60, 61, 62] and even for anisotropic plasmas [63], showing a combination of near-isothermal and polytropic behaviors.

A key point is that, if an auxiliary kinetic model is used (and collisionality is weak), a closure of the fluid equations at the level of the momentum equation is the most convenient. Thus, the proposed electron fluid model consists of the following equations:

$$
\begin{aligned}
& \frac{\partial \rho_{c}}{\partial t}+\nabla \cdot \boldsymbol{j}=0 \\
& 0=-\nabla \cdot \mathcal{P}_{e}-e n_{e}\left(-\nabla \phi+\boldsymbol{u}_{e} \times \boldsymbol{B}\right)-\sum_{s=1}^{L} v_{e s} m_{e} n_{e}\left(\boldsymbol{u}_{e}-\boldsymbol{u}_{s}\right) \\
& \nabla^{2} \phi=-\frac{\rho_{c}}{\epsilon_{0}}
\end{aligned}
$$

where the charge and electric current densities satisfy

$$
\begin{gathered}
\rho_{c}=e\left(n_{e}^{*}-n_{e}\right), \quad n_{e}^{*}=\sum_{s=1}^{L} Z_{s} n_{s} \\
j=j_{i}+j_{e}, \quad j_{i}=e \sum_{s=1}^{L} Z_{s} n_{s} \boldsymbol{u}_{s}, \quad \boldsymbol{j}_{e}=-e n_{e} \boldsymbol{u}_{e}
\end{gathered}
$$


In addition, $n_{s}$ and $\boldsymbol{u}_{s}$ are the fluid velocity of the $s^{\text {th }}$ heavy species population, obtained from the PIC model, $u_{e}$ is the electron fluid velocity, $n_{e}^{*}$ is the quasineutral electron density, and $v_{e s}$ is the electron momentum transfer collision frequency with population $s$ (refer to Appendix A for more details).

For the purposes of the present paper, we will limit the electron fluid model to an unmagnetized plume $(B=0)$ and to the simple polytropic electron closure:

$$
\mathcal{P}_{e}=p_{e} \mathcal{I} \quad \text { with } \quad p_{e}\left(n_{e}\right)=n_{e} T_{e}, \quad T_{e}=T_{e 0}\left(\frac{n_{e}}{n_{e 0}}\right)^{\gamma-1}
$$

where $\gamma$ is the constant polytropic coefficient, and $n_{e 0}, T_{e 0}$ are the electron density and temperature at the plume location where we have set $\phi=0$. Thus, Eq. 13 becomes

$$
0=-\nabla p_{e}+e n_{e} \nabla \phi+\frac{m_{e} v_{e}}{e}\left(j-j_{d}\right)
$$

where

$$
v_{e}=\sum_{s=1}^{L} v_{e s}, \quad j_{d}=j_{i}-\frac{e n_{e}}{v_{e}} \sum_{s=1}^{L} v_{e s} \boldsymbol{u}_{s}
$$

are the total electron collision frequency and a driving current density (it shall appear as a forcing term in the final differential equation), respectively. Solving the momentum equation for $\boldsymbol{j}$, the generalized Ohm law is finally obtained:

$$
j=\frac{\sigma_{e}}{e} \nabla H_{e}+j_{d}
$$

where $\sigma_{e}=e^{2} n_{e} / v_{e} m_{e}$ is the electron conductivity,

$$
H_{e}=h_{e}-e \phi
$$

is the electron Bernoulli function, and

$$
h_{e}\left(n_{e}\right)= \begin{cases}T_{e 0} \ln \left(\frac{n_{e}}{n_{e 0}}\right), & \gamma=1 \\ -\frac{\gamma T_{e 0}}{(\gamma-1)}\left[1-\left(\frac{n_{e}}{n_{e 0}}\right)^{\gamma-1}\right], & \gamma>1\end{cases}
$$

is the barotropic function, satisfying $\nabla h_{e}=\nabla p_{e} / n_{e}$. Notice that we have set $h_{e}, H_{e}=0$ at the location where $\phi=0$.

Introducing now Eq. 20, into Eq. 12, an elliptic differential equation for $H_{e}$ is obtained:

$$
\nabla^{2} H_{e}+\nabla \ln \sigma_{e} \cdot \nabla H_{e}=-\frac{e}{\sigma_{e}}\left(\nabla \cdot j_{d}+\frac{\partial \rho_{c}}{\partial t}\right)
$$

Therefore, the fluid model reduces basically to solving two coupled elliptic equations: the above one for $H_{e}$ and Poisson equation for $\phi$. Typical boundary conditions set either these magnitudes or the derivatives perpendicular to the walls. In the case of the Bernoulli function, the perpendicular derivative is indeed a condition on the electric current density:

$$
\frac{\partial H_{e}}{\partial \mathbf{1}_{\perp}}=\frac{e}{\sigma_{e}}\left(j-j_{d}\right) \cdot \mathbf{1}_{\perp},
$$

where $1_{\perp}$ is the unit vector normal to a considered boundary and directed towards the plasma. 


\section{The quasineutral closure}

Let us consider first the zero Debye length limit, for which the whole plume can be considered quasineutral. The mathematical structure of the electron problem simplifies. First of all, Poisson equation reduces to

$$
n_{e}=n_{e}^{*} \quad\left(\text { i.e. } \quad \rho_{c}=0\right)
$$

which determines the electron density and, subsequently, the electron temperature $T_{e}^{*}=$ $T_{e}\left(n_{e}^{*}\right)$, the barotropic function $h_{e}^{*}=h_{e}\left(n_{e}^{*}\right)$, the conductivity $\sigma_{e}^{*}=\sigma_{e}\left(n_{e}^{*}\right)$, and the driving current $\boldsymbol{j}_{d}^{*}=\boldsymbol{j}_{d}\left(n_{e}^{*}\right)$. Notice that all these quantities are a function of the PIC model solution. The uncoupled equation for the Bernoulli's function then simplifies to:

$$
\nabla^{2} H_{e}+\nabla \ln \sigma_{e}^{*} \cdot \nabla H_{e}=-\frac{e}{\sigma_{e}^{*}} \nabla \cdot j_{d}^{*}
$$

Once the solution for $H_{e}$ is obtained, $\boldsymbol{j}$ is given by the generalized Ohm equation, Eq. 20, and the electric potential from Eq. 21, that is:

$$
\phi=\frac{h_{e}-H_{e}}{e}
$$

Notice that the widely-used isothermal and polytropic models, leading to Boltzmann-like relations, correspond to setting $H_{e} \equiv 0$ in Eq. 26, which is the solution in collisionless limit $\sigma_{e}^{*} \rightarrow \infty$ of Eq. 25. Furthermore, in this limit, the electric current in the generalized Ohm law, Eq. 20, is indefinite, which is the most severe limitation of the Boltzmann relation/polytropic models. The present weakly-collisional model overcomes that limitation, by computing the contribution of $\sigma_{e} \nabla H_{e} / e$ to the net electric current. The simulations of Sec. 7 will show that this contribution is central to study the plasma plume neutralization (at the exit of GITs or HETs, for instance), and the plume-S/C interaction.

\subsection{The sheath model}

In the quasineutral closure, infinitely-thin Debye sheaths are postulated between the quasineutral solution and the walls, in order to accommodate potentials and electric currents there. Thus, at such boundaries, we must distinguish between the wall potential $\phi_{W}$, and the potential at the sheath edge $\phi_{S}$ (of the quasineutral solution). The sheath model then establishes a relation between these two potentials and the perpendicular current density to the wall $j_{W}=j \cdot \mathbf{1}_{\perp}=j_{i, W}+j_{e, W}$ (positive if emitted by the wall, for $\mathbf{1}_{\perp}$ oriented towards the plasma).

In the most common case of a "negative" sheath, it is $\phi_{W}<\phi_{S}$, in order to confine electrons. The well-known solution for a negative planar (and collisionless) sheath, assuming a Maxwellian-like electron distribution at its edge, relates $\phi_{W}$ and $j_{W}$ through:

$$
\phi_{W}=\phi_{S}-\frac{T_{e}}{e} \ln \left(\frac{\left(j_{W}-j_{i, W}\right)}{e n_{e}} \sqrt{\frac{2 \pi m_{e}}{T_{e}}}\right)
$$


where the ion current density, the electron density $n_{e}$ and temperature $T_{e}$ refer to the sheath edge.

In the case of a dielectric wall, the net-current free condition $j_{W}=0$ yields $\phi_{W}$ locally. This is also the case of current-emitting walls (by thermoemission, photoemission, thermionic emission) where $j_{W}$ is known locally from the PIC model solution (or from known wall heating/illumination conditions).

For conductive walls (being them emissive or not), the problem is more complex, since the boundary condition is non-local. Typically, from the knowledge of the electric potential $\phi_{W}$, we compute the total electric current to a conductive wall (or object) as

$$
I_{W}=-\sum j_{W} \Delta S
$$

where the summation extends over the material boundary faces of the conductive object, and $j_{W}$ satisfies Eq. 27.

Furthermore, conductive walls can present locally positive sheaths, i.e. locations with $\phi_{W}>\phi_{S}$. In these locations, the current density is approximated by

$$
j_{W} \simeq j_{i, W}-e n_{e} \sqrt{T_{e} /\left(2 \pi m_{e}\right)} .
$$

For a positive planar sheath, the electron current thus equals the thermal flux in the plasma, and the PIC model must take into account the deceleration and the eventual reflection of the low energy ions within the sheath, as already discussed in Sec. 2.3.1.

\subsection{The equivalent circuit solver}

The presence, in a typical plume-SC interaction, of several conductive walls with nonlocal boundary conditions complicates the integration of the time-dependent equations. As done in other existing plume-spacecraft interaction codes [27], an efficient way to overcome that difficulty is to add an equivalent electric circuit linking the different walls of the problem and the plasma. Fig. 4 illustrates this numerical scheme. Each conductive object $l$ is assumed to be an iso-potential node, receiving a current from the plasma $I_{W, l}$ (indeed Eq. 28) and connected to another iso-potential node $m$ by means of both a resistance $R_{l m}$, and a forced voltage bias $V_{l m}$.

One of the nodes (the S/C node in Fig. 4) is considered as independent and its potential relative to the plasma reference point (with $\phi=0$ ) is first computed as the time evolution of the voltage of an equivalent capacitor $C$,

$$
\frac{d \phi_{1}}{d t}=\frac{I}{C}
$$

where the charging current $I$ is obtained as the sum of the plasma currents to the circuit nodes $I=\sum_{l} I_{W, l}$. In the steady state, $I$ must be zero and $\phi_{1}$ is the floating potential of the S/C. Kirchhoff laws then yield:

$$
\begin{cases}\text { current continuity at dependent node } l: & I_{W, l}+\sum_{m \neq l} I_{m l}=0 \\ \text { potential across connection } l m: & \phi_{l}-I_{l m} R_{l m}+V_{l m}-\phi_{m}=0\end{cases}
$$




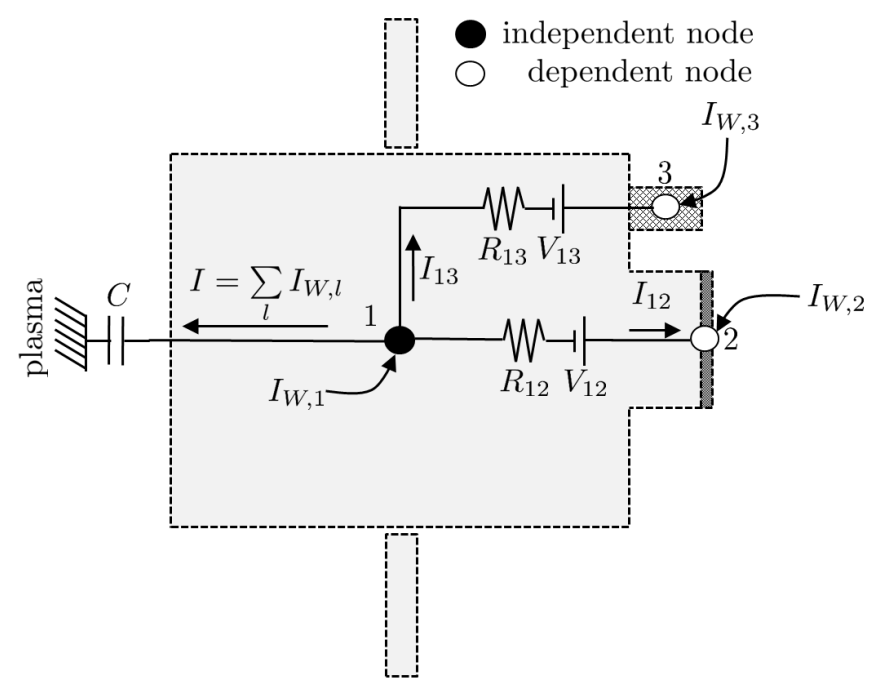

Figure 4: Scheme of the equivalent circuit, for a simulation featuring 3 different conductive objects.

For the case of Fig. 4, there are 2 dependent nodes and 2 connections. Eqs. 30 thus determine the 2 unknown dependent node potentials and internode currents. Values of $R_{l m}, V_{l m}$ and $C$ are selected based on either real electrical properties of the $\mathrm{S} / \mathrm{C}$ parts or to facilitate numerical convergence.

\section{Treatment of non neutral regions}

Once the quasineutral solution for the electron density $n_{e}^{*}$, the electric potential $\phi^{*}$, and the electron temperature $T_{e}^{*}$ are known at the instant $t^{(k)}$, the simulation domain is dynamically split into quasineutral and non-neutral subdomains, and the solution at time $t^{(k)}$ is recomputed in the non-neutral subdomain.

First of all, the quasineutral subdomain, constituted by quasineutral nodes and boundary faces must be defined. We will consider that cells and boundary faces are quasineutral if their level of non-neutrality is below a maximum value, called $\varepsilon_{\text {max }}$ (a value of 0.032 is considered later in simulations, which corresponds to a relative charge density of $1 \%$ ). For inner cell nodes, non-neutrality is measured by

$$
\varepsilon_{n}=\left|\frac{\epsilon_{0} \nabla^{2} \phi^{*}}{e n_{e}^{*}}\right|^{1 / 2}=\left|\frac{n_{e}^{*}-n_{e}}{n_{e}^{*}}\right|^{1 / 2}
$$

and the cell node is part of the non-neutral subdomain when $\varepsilon_{n}>\varepsilon_{\text {max }}$. For boundary faces, non-neutrality is measured by the ratio between the local Debye length and the cell size $\Delta l$ (in the direction normal to the surface):

$$
\varepsilon_{f}=\frac{1}{\Delta l} \sqrt{\frac{\epsilon_{0} T_{e}^{*}}{e^{2} n_{e}^{*}}}
$$

Now it must be decided whether a discontinuity sheath is postulated at the boundary of the simulation domain (which we will call $\mathrm{S}$ ) or this boundary is already the wall W. Numerical 
convergence between these two cases demands to add an intermediate case. Therefore, three cases are considered:

- if $\varepsilon_{f} \geq 1$ : the boundary face is non-neutral, the sheath is directly simulated inside the plasma domain, and the boundary face is the wall, i.e. $\phi_{S}=\phi_{W}$,

- if $\varepsilon_{f} \leq \varepsilon_{\text {max }}$ : the boundary face is quasineutral, the sheath is treated separately as a discontinuity, and the potential at $\mathrm{S}$ is the quasineutral sheath edge potential $\phi_{S}^{*}$,

- if $\varepsilon_{\max }<\varepsilon_{f}<1$, a partial sheath is added outside of the plasma domain, with a potential drop given by:

$$
\phi_{S}-\phi_{W}=\frac{1-\varepsilon_{f}}{1-\varepsilon_{\max }}\left(\phi_{S}^{*}-\phi_{W}\right)
$$

where $\phi_{S}^{*}$ and $\phi_{W}$ are known from respectively the quasineutral solution and the current wall potential.

We point out that for computational purposes, Eqs. 31 and 32 are always estimated at the mesh nodes. Once the non-neutral subdomain is defined, Poisson equation, Eq. 36, and Eq. 23 for the Bernoulli function should be integrated simultaneously to determine $\phi$ and the electron fluid magnitudes at these mesh nodes. This is particularly costly computationaly, so a different approach is proposed here. First, we assume that $\partial \rho_{c} / \partial t \ll \nabla \cdot \boldsymbol{j}_{d}$ (i.e. we focus on quasi-steady solutions) and we propose to obtain $H_{e}$ from Eq. 25 with the substitutions

$$
\sigma_{e}^{*} \rightarrow \sigma_{e}^{(k-1)}, \quad j_{d}^{*} \rightarrow j_{d}^{(k-1)}
$$

Then, from Eqs.21 and 22, one has (for the generic polytropic case)

$$
n_{e}\left(H_{e}, \phi\right)=n_{e 0}\left[1+\frac{\gamma-1}{\gamma} \frac{H_{e}+e \phi}{T_{e 0}}\right]^{\frac{1}{\gamma-1}}
$$

which substituted into Poisson equation, yields a nonlinear equation for $\phi$,

$$
\frac{\epsilon_{0}}{e} \nabla^{2} \phi=n_{e}\left(H_{e}, \phi\right)-n_{e}^{*}
$$

The numerical procedure to solve this equation is explained in Appendix B.

\section{The overall simulation loop}

A generic step of the overall simulation loop is shown in Fig. 5, for a general non-neutral simulation. The particle push represents several PIC algorithms and generates updated fluid properties at times $k+1$ (quasineutral electron density $n_{e}^{*}$, and heavy particle densities $n_{s}$ ) and $k+1 / 2$ (heavy particle fluid velocities $\boldsymbol{u}_{s}$, and surface weighted variables at material boundaries). The quasineutral electron density and the surface weighted $P$ variable are then fed to the Bohm condition forcing algorithm, which corrects, if need be, the value of the electron density at the quasineutral material boundaries, thus completing the PIC sub-step.

Then, boundary conditions for the fluid closure at this new time $k+1$ need to be updated. First, the sheath solver updates the electron current density $j_{e, W}$ at conductive walls, and 


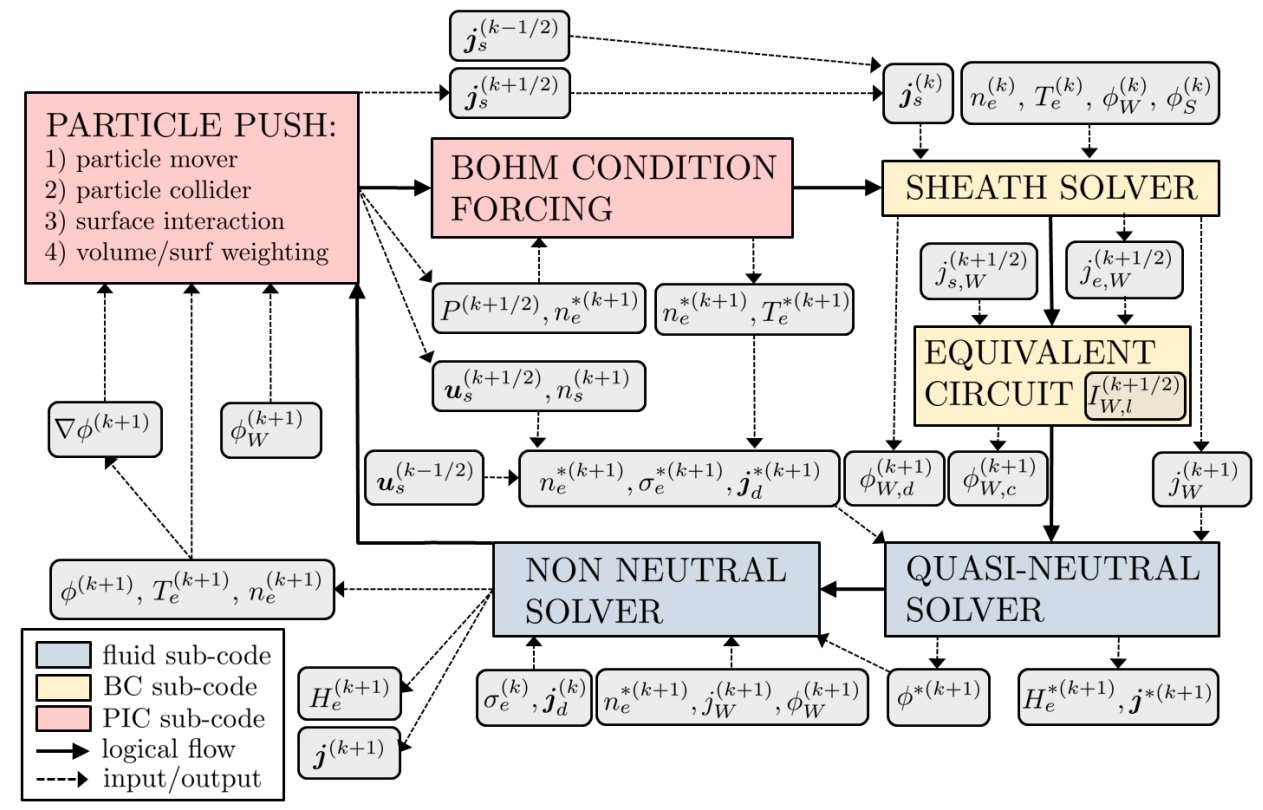

Figure 5: The hybrid-particle code simulation loop, at the time step $k+1$ (from time $t^{(k)}$ to time $\left.t^{(k+1)}\right)$

the dielectric wall potential $\phi_{W, d}$ both at time $k$, since its inputs (wall potential, sheath edge potential, electron density, temperature, and ion current) are known at this time step. The electron current density is then extrapolated to time $k+1 / 2$ and passed together with the ion current density to the wall $j_{i, W}$ (at time $k+1 / 2$ ) to the equivalent circuit solver. This obtains the electric current $I_{W, l}^{(k+1 / 2)}$ to the conductive objects and updates their potentials to time $k+1$, with a second-order leap-frog scheme. The sheath solver also extrapolates the dielectric walls potential and the total electric current density to the wall $j_{W}=j_{e, W}+j_{i, W}$ to time $k+1$, as needed by the fluid closure algorithms.

This is then fed, together with the quasineutral electron density $n_{e}^{*}$, conductivity $\sigma_{e}^{*}$, and driving current density $\boldsymbol{j}_{d}^{*}$, all at time $k+1$, to the quasineutral solver, which solves for the quasineutral electric potential $\phi^{*}$, the Bernoulli function $H_{e}^{*}$, and the electric current density $j^{*}$ at time $k+1$. The non neutral solver then receives as input the quasineutral potential $\phi^{*}$, the wall potential $\phi_{W}$, and the quasineutral density $n_{e}^{*}$ at time $k+1$, and assumes the latest step values for $\sigma_{e}$ and $\boldsymbol{j}_{d}$. Its outputs are the electric potential $\phi$, the electron temperature $T_{e}$, density $n_{e}$ and Bernoulli function $H_{e}$ at the time step $k+1$. These, together with the updated wall potentials at time $k+1$ are fed back to the PIC model and the next time step is finally initiated.

The exact time consistency described above permits achieving a second-order accuracy in the time integration. However, a commonly used approach, that helps reduce the numerical noise and is considered in the simulations of Sec. 7, is to use time-averaged properties at the material boundaries (as already mentioned in Sec. 2.3.2), with a number of averaging steps in the order of 50-100 ( $\Delta k_{\text {avg }}=100$ in the presented simulations). 


\section{Simulations}

\subsection{Definition of the simulation geometry and settings}

The plume-S/C interaction scenario considered for the model validation features a cubic $\mathrm{S} / \mathrm{C}$ with an ion thruster, a hollow cathode neutralizer, and two solar arrays, as shown in Fig. 6 (a) and (b), while the corresponding equivalent circuit was already shown in Fig. 4.

(a)

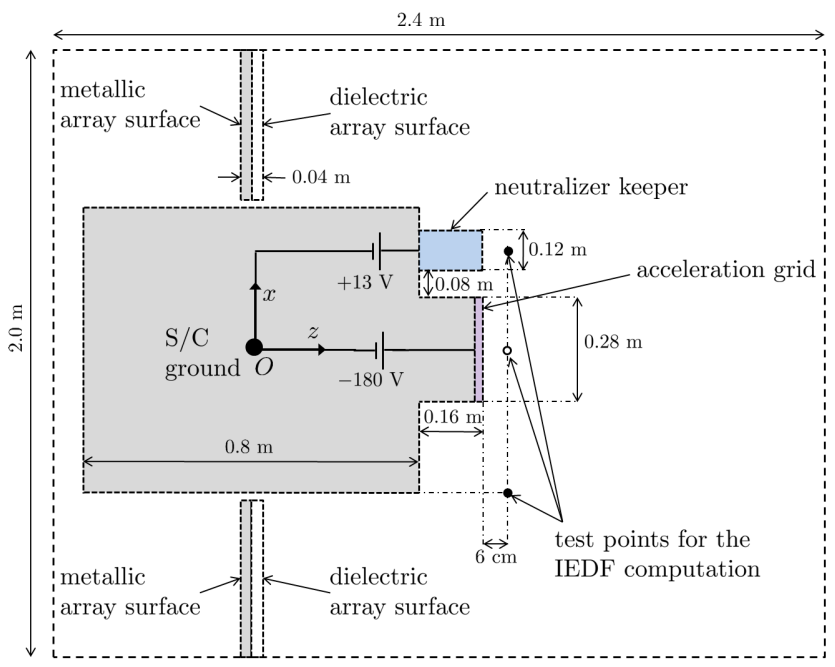

(b)

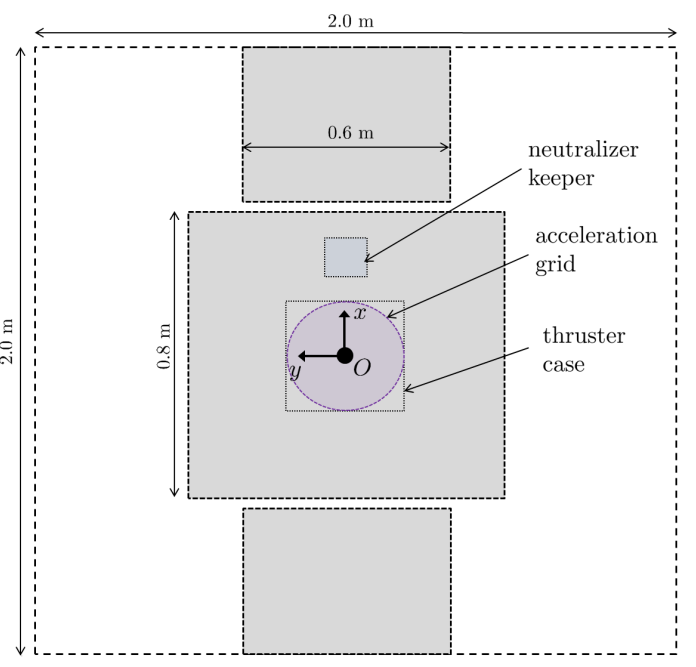

Figure 6: Simulation domain for the spacecraft-plume interaction simulation: (a) $x-z$ crosssection, and (b) $x-y$ cross-section, both through the satellite center. The PIC mesh is Cartesian, with a number of nodes along $x, y$, and $z$ of $101 \times 101 \times 121(2 \mathrm{~cm}$ side cells $)$. The white dot on the thruster symmetry axis is the reference point for the electron properties, the electric potential, and the Bernoulli function. Two additional points on the thruster side and on the neutralizer axis are considered for the computation of the ion energy distribution function.

Three conductive objects are considered: the satellite ground (including cubic body, thruster case and the back-face of the solar arrays), the neutralizer keeper external surface, and the most external grid of the thruster. The resistances between $\mathrm{S} / \mathrm{C}$ ground and acceleration grid and neutralizer keeper are assumed to be zero $\left(R_{12}=R_{13}=0\right)$. The front face of the solar arrays, as shown in Fig. 6 (a), is modeled as a dielectric object (i.e. the wall potential is determined locally from current equilibrium).

The values of some simulation parameters are summarized in Tab. 3. The considered thruster simulates the NASA's NSTAR ion thruster [64, 65], while the applied voltages to the acceleration grid and neutralizer keeper are kept constant to respectively $V_{12}=-180$ and $V_{13}=+13 \mathrm{~V}$. The negative grid potential prevents most of electrons from backstreaming towards it, while the neutralizer keeper is biased positive with respect to the spacecraft ground [65].

The injection areas for both the thruster and neutralizer are circular with radii of respectively 14 and $4 \mathrm{~cm}$. Neutrals are injected axially with sonic conditions and with a flat density profile from both the thruster and neutralizer injection cells. Regarding the thruster ions, these are injected following a Self Similar profile [13, 17], with an outermost streamline 
Table 3: Considered parameters for the plasma-plume satellite interaction simulation. Applied voltages refer to the spacecraft ground. All considered objects are conductive, except for the front surface of the solar arrays, which is dielectric.

\begin{tabular}{|c|c|c|}
\hline Simulation parameter & Units & Values \\
\hline Neutralizer keeper voltage & $\overline{\mathrm{V}}$ & +13 \\
\hline Acceleration grid voltage & $\mathrm{V}$ & -180 \\
\hline Thruster mass flow rate & sccms & 27.13 \\
\hline Thruster mass utilization efficiency & $\%$ & 90.0 \\
\hline Doubly to singly charged ion current ratio & $\%$ & 9.1 \\
\hline Injected $\mathrm{Xe}^{+}$profile (thruster) & $\mathrm{n} / \mathrm{a}$ & $\begin{array}{c}\operatorname{SSM}\left(R_{0}=14 \mathrm{~cm}, \text { and }\right. \\
\left.\alpha_{0}=20.5 \mathrm{deg}\right)\end{array}$ \\
\hline Injected $\mathrm{Xe}^{++}$profile(thruster) & n/a & $\begin{array}{c}\operatorname{SSM}\left(R_{0}=14 \mathrm{~cm}, \text { and }\right. \\
\left.\alpha_{0}=30 \mathrm{deg}\right)\end{array}$ \\
\hline Injected $\mathrm{Xe}^{+}$energy (thruster) & $\mathrm{eV}$ & 1040 \\
\hline Injected $\mathrm{Xe}^{++}$energy (thruster) & $\mathrm{eV}$ & 2080 \\
\hline Injected $\mathrm{Xe}^{+}$temperature (thruster) & $\mathrm{eV}$ & 0.1 \\
\hline Injected $\mathrm{Xe}^{++}$temperature (thruster) & $\mathrm{eV}$ & 0.2 \\
\hline Injected neutrals profile (thruster) & $\mathrm{n} / \mathrm{a}$ & Flat \\
\hline Injected neutrals velocity (thruster) & $\mathrm{m} / \mathrm{s}$ & 247 (sonic) \\
\hline Injected neutrals temperature(thruster) & $\mathrm{eV}$ & 0.05 \\
\hline Neutralizer mass flow rate & sccms & 3.59 \\
\hline Injected neutrals profile (neutralizer) & $\mathrm{n} / \mathrm{a}$ & Flat \\
\hline Injected neutrals velocity (neutralizer) & $\mathrm{m} / \mathrm{s}$ & 247 (sonic) \\
\hline Injected neutrals temperature (neutralizer) & $\mathrm{eV}$ & 0.05 \\
\hline Neutralizer ion flow percentage & $\%$ & 5.0 \\
\hline Injected $\mathrm{Xe}^{+}, \mathrm{Xe}^{++}$profile (neutralizer) & $\mathrm{n} / \mathrm{a}$ & $\begin{array}{l}\text { Thermal, Gaussian } \\
\qquad\left(R_{0}=4 \mathrm{~cm}\right)\end{array}$ \\
\hline Injected $\mathrm{Xe}^{+}$temperature (neutralizer) & $\mathrm{eV}$ & 0.2 \\
\hline Injected $\mathrm{Xe}^{++}$temperature (neutralizer) & $\mathrm{eV}$ & 0.4 \\
\hline Electron temperature at thruster exit & $\mathrm{eV}$ & 3.5 \\
\hline Electron polytropic cooling coefficient & $\mathrm{n} / \mathrm{a}$ & {$[1.0,1.3]$} \\
\hline
\end{tabular}

divergence angle $\alpha_{0}=20.5 \mathrm{deg}$ (at the radius $R_{0}=14 \mathrm{~cm}$ from the thruster centerline). This divergence profile corresponds to a divergence efficiency of 0.98, as considered in Ref. [65]. An already developed divergence angle is considered because the mesh is not fine enough to simulate the effects of beamlets injection and coalescence into a single beam, so that the injection surface is actually simulated as a quasineutral surface, with a thin sheath (across which the potential drops from its value at the quasineutral plasma to that of the acceleration grid). For what concerns the neutralizer ions, past studies [66] have shown that a significant ion current is also emitted. Here we have considered that $5 \%$ of the total mass flow of the neutralizer is emitted in the form of singly or doubly charged ions from a thermal reservoir (with temperature of respectively 0.2 and $0.4 \mathrm{eV}$ ). Finally, a ratio between doubly and singly charged ion current of $9.1 \%$ [67] has been considered for both the thruster and the neutralizer emissions. 
Regarding the electron thermodynamics, a peak electron temperature of $3.5 \mathrm{eV}$, consistent with existing experimental measurements for similar thrusters [68], is assumed at a node located $6 \mathrm{~cm}$ downstream from the thruster exit (which represents the reference plasma point for potential, Bernoulli function and electron enthalpy), while four different values for $\gamma$ are considered: 1.0 (isothermal), 1.1 (reference case), 1.2 and 1.3.

For what concerns the applied fluid closure, the simulations are run with the sole quasineutral solver between $t=0$ and $t=1 \mathrm{~ms}$, and, starting from $t=1 \mathrm{~ms}$, the nonneutral solver is activated. The requested time for a slow CEX ion (with an energy content of $5 \mathrm{eV}$ ) to cross the entire simulation domain is around $1 \mathrm{~ms}$, so that the considered simulation time is expected to be sufficient to reach stationary conditions.

The boundary conditions for the computation of the Bernoulli function $H_{e}$, for this simulation setup are:

- Current free condition $\boldsymbol{j} \cdot \mathbf{1}_{\perp}=0$ at the external boundaries and at the dielectric walls of the solar arrays front surface, thus yielding:

$$
\frac{\partial H_{e}}{\partial 1_{\perp}}=-\frac{e}{\sigma_{e}} j_{d} \cdot \mathbf{1}_{\perp}
$$

- Fixed electric current $j_{W}=j_{W}\left(\phi_{W}\right)$ to the other conductive walls:

$$
\frac{\partial H_{e}}{\partial \mathbf{1}_{\perp}}=\frac{e}{\sigma_{e}}\left(j_{W}-j_{d} \cdot \mathbf{1}_{\perp}\right)
$$

- Dirichlet conditions $H_{e}=$ const, at the emissive surface of the neutralizer, equivalent to leaving a free electron current to balance the electron current lost to the boundaries, and generated inside the domain (e.g. due to ionization).

Regarding the electric potential, the boundary conditions (already discussed above) are:

- Dielectric or conductive wall potential $\phi=\phi_{W}$ at the non-neutral material boundary nodes.

- Transition conditions $\phi=\phi_{S}$ (refer to Eq. 33) at the transition material boundary nodes.

- Quasineutral electric potential $\phi=\phi^{*}$ at all quasineutral nodes (including those on the external boundary).

- $\phi=0$ at the reference node for the electron properties $(6 \mathrm{~cm}$ downstream from the thruster exit area, on its axis).

- Neumann conditions on $\phi$ at the non-neutral external boundary nodes:

$$
\frac{\partial \phi}{\partial \mathbf{1}_{\perp}}=0 .
$$

\subsection{Discussion of the simulation results}

The simulation results for the reference simulation case $(\gamma=1.1)$ are shown in Fig. 7 (a) to (h). The electric potential is shown in Fig. 7 (a). The $\mathrm{S} / \mathrm{C}$ is floating at a potential of $-38 \mathrm{~V}$ with respect to the plasma plume near the thruster exit (the reference potential point is shown 
by a white dot), so that the iso-potential lines adapt to this value close to the cubic S/C body, through spatially resolved plasma sheaths. The effect of the CEX ions is clearly visible on the left of the main plasma plume, while the neutralizer creates a plasma bridge with the thruster (necessary for the plume current neutralization) and alters substantially the symmetry of the plasma response.

The electron density is shown in Fig. 7 (b), and follows closely the evolution of the electric potential, with densities rapidly dropping to zero as the very negative S/C surfaces are approached. On the lateral sides of the thruster, the electron density nearly coincides with the CEX ion density and shows values between $10^{12} \mathrm{~m}^{-3}$ and $10^{13} \mathrm{~m}^{-3}$, a result which is consistent with the simulation results and experimental measurements of past studies of the same thruster, like those of Refs. [69], [70], and [71].

The electric current density is shown in Fig. 7 (c). A virtually current-free plasma plume is achieved just $30-40 \mathrm{~cm}$ downstream from the thruster exit, which is consistent with the assumption that the far-region plasma is essentially current free. All electric current streamlines originate at the thruster exit (due to the emitted ions) and reach the neutralizer emission surface (due to emitted electrons) just as expected (no electric current sources exist inside the domain). The total electric current is given by Ohm law, Eq. 20, and presents two different contributions: the product $\sigma_{e} \nabla H_{e} / e$ and the driving current $j_{d}$. The clearly dominating term is the former, as shown in Fig. 7 (d), showing its relative relative magnitude (normalized with the electric current density) and streamlines. In fact, although the absolute potential correction $H_{e}$ is almost negligible (the plasma is lowly collisional) and in the order of a few $\mathrm{mV}$, the electric conductivity is quite high (ranging from $3000 \Omega^{-1} \mathrm{~m}^{-1}$ inside the main plume, to $1000 \Omega^{-1} \mathrm{~m}^{-1}$ at the neutralizer exit, and $500-1000 \Omega^{-1} \mathrm{~m}^{-1}$ in the lateral plume regions populated by CEX ions). The only region in which the driving term $j_{d}$ is comparable in magnitude to $\sigma_{e} \nabla H_{e} / e$ is the central region of the main plasma plume and accounts for approximately half of the total electric current density.

Regarding the simulation domain sub-division into quasineutral and non-neutral regions, this is shown in Fig. 7 (e), showing the normalized charge density ( $\epsilon_{n}^{2}$ in Eq. 31). The white regions correspond to the quasineutral subdomain, featuring a normalized charge density (in absolute value) lower than $1 \%$. Regarding the S/C walls, these are all non-neutral, with the injection areas of the thruster and neutralizer being the only quasineutral boundary surfaces. The difference in potential due to the solution of the non-neutral regions (with the Poisson solver) is finally shown in Fig. 7 (f). The largest differences between the non-neutral and neutral solver solutions are found close to the S/C surfaces and reach values around $10 \mathrm{~V}$ (with the non neutral solution being more negative).

An important capability of a S/C-plasma interaction tool is to predict the ion current flux to the $\mathrm{S} / \mathrm{C}$ walls, and the ion mean wall-impact energy (to evaluate the effects of sputtering and deposition). Fig. 7 (g) shows the ion current density reaching the S/C front surfaces (the absolute value of $j_{i, W}$ ). A maximum ion current density of up to $10 \mathrm{~mA} \mathrm{~m}^{-2}$ is reached at the corners of the thruster case and on one side (the one closer to the thruster) of the neutralizer keeper. The average current density to the $\mathrm{S} / \mathrm{C}$ cubic body is around $0.5 \mathrm{~mA} \mathrm{~m}^{-2}$. The current density to the solar array, on the other hand, is generally lower than $0.1 \mathrm{~mA} \mathrm{~m}^{-2}$. Fig. 7 (h) 

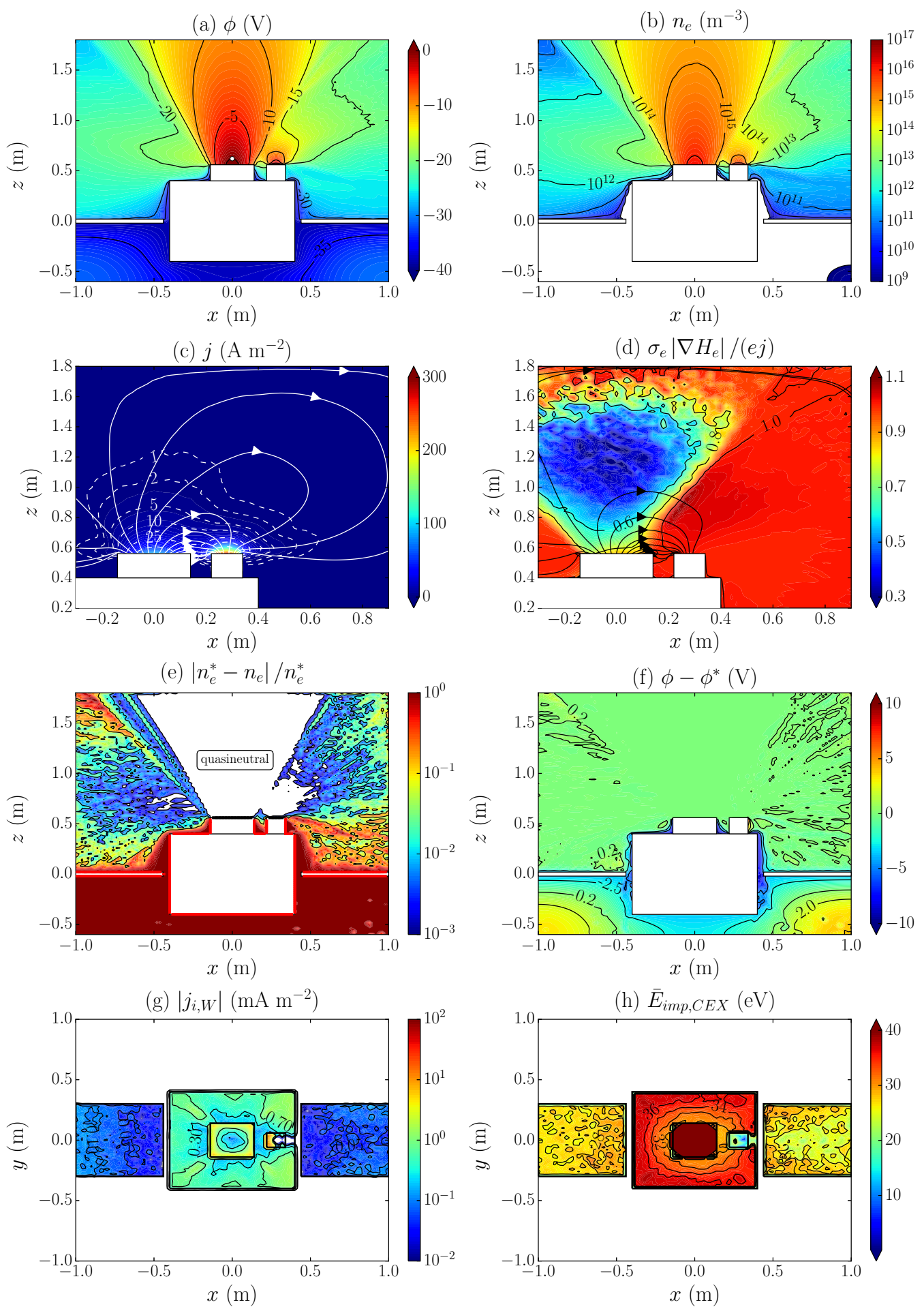

Figure 7: Simulation results for the reference case with $\gamma=1.1$ : (a) electric potential (reference point is shown with a white dot), (b) electron density, (c) electric current density and streamlines, (d) relative contribution of $\sigma_{e} \nabla H_{e} / e$, (e) non neutrality ratio or $\epsilon_{n}^{2}$, (f) difference on the electric potential between the non-neutral and quasineutral solvers, (g) total ion current density to the S/C front walls, and (h) average ion wall-impact energy on the S/C front walls. Subplots (a) to (f) refer to the $y=0$ cross section, while subplots (g) and (h) to the front surfaces of thruster case, neutralizer, S/C cubic body and solar arrays. 
finally shows the average wall-impact kinetic energy of the ions hitting the $\mathrm{S} / \mathrm{C}$ walls. This is in the order of 30-35 eV at the S/C cubic body front surface, and lower than $30 \mathrm{eV}$ at the solar arrays. These values depend mostly on the floating potential of the $\mathrm{S} / \mathrm{C}$ with respect to the plasma plume denser region (where most of CEX ions are generated).

A quite interesting feature are the three dimensional streamlines of the electron current density, which are shown in Fig. 8. The farthest (from the neutralizer) conical plume streamlines are neutralized by electrons that circle around the dense region of the plume. Moreover, all electron streamlines originate from the emission surface of the neutralizer keeper, because $\nabla \cdot j_{e} \approx 0$ inside this lowly collisional plasma (ionization effects are almost negligible).

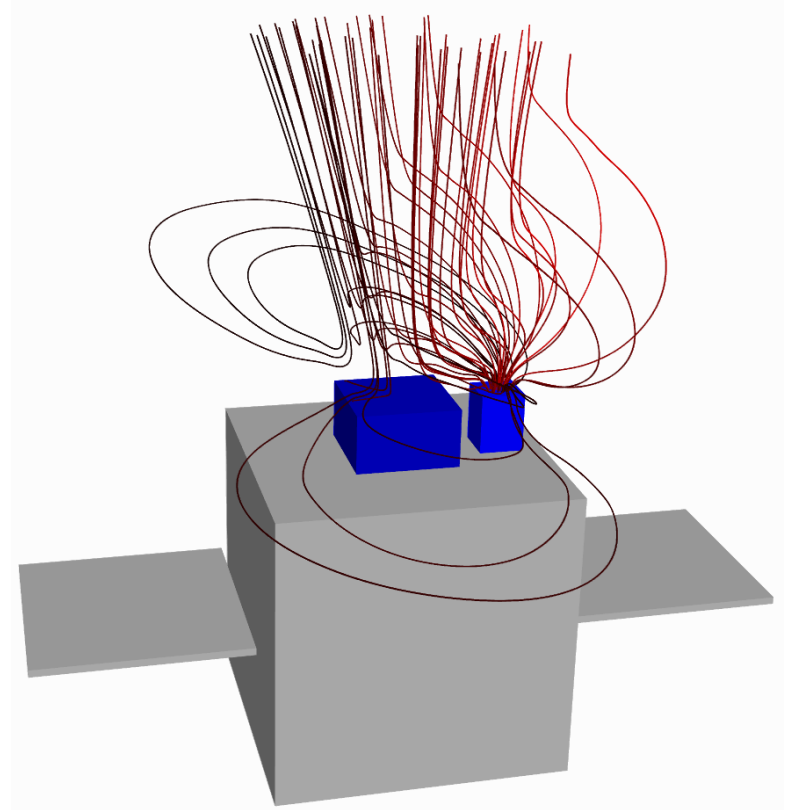

Figure 8: Three-dimensional electron streamlines emanating from the neutralizer and merging with the ion plume. The farther (from the neutralizer) the ion streamline to be neutralized, the darker the color of the corresponding electron streamline.

The ion energy distribution function [72] at the three test points of Fig. 6 (a), is shown in Fig. 9 (a). Close to the thruster exit (black solid line), three well defined populations of ions can be identified: the emitted doubly charged ions (with energies around $2080 \mathrm{eV}$ ), the emitted singly charged ions (with energies around $1040 \mathrm{eV}$ ), and the slow CEX ions (including both singly and doubly charged ions), which feature energies that are generally below $3 \mathrm{eV}$. On the thruster side (dotted blue line), on the other hand, only CEX ions are present, and the singly and doubly charged ions populations can be distinguished again in terms of energy content. In fact the energy of these ions depends directly on the potential difference between the location where they are generated (dense plume region, with $\phi \in[0,-5] \mathrm{V}$ ) and the considered point potential $(\phi \approx-20 \mathrm{~V})$. Finally, at the neutralizer axis, the emitted singly and doubly charged ions can be clearly distinguished from CEX ions generated in the plume: the two well definite peaks at energies of approx. 15 and $30 \mathrm{eV}$ are indeed caused by the acceleration of the emitted ions, due to the ambipolar electric field at the neutralizer exit (characterized by a 
steep potential drop). Finally, a zoom of the ion energy distribution function at the thruster axis is shown in Fig. 6 (b), which also provides the separate contributions of singly and doubly charged ions. The disparity of energies and distributions of the different ion populations shown in Fig. 9 (a) and (b) clearly makes the use of multiple fluids for the ion species quite troublesome.

(a)

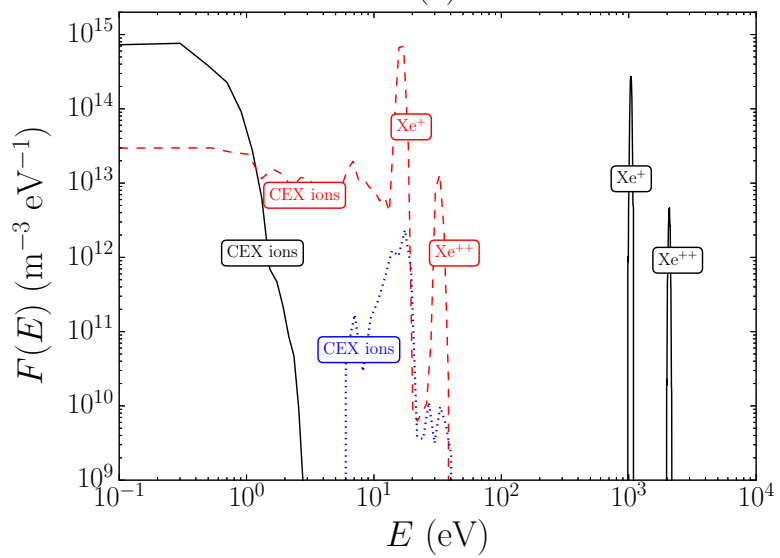

(b)

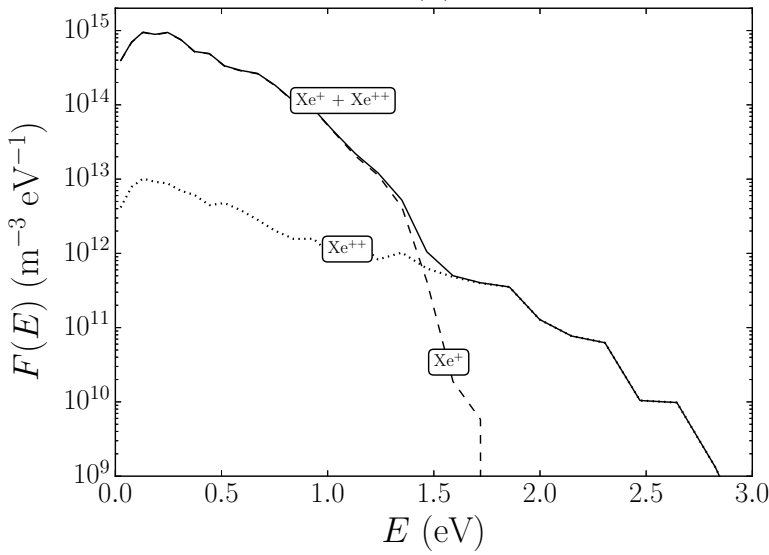

Figure 9: (a) Ion energy distribution function at three different locations, shown in Fig. 6: thruster axis (black solid line), neutralizer axis (red dashed line), and $40 \mathrm{~cm}$ off the thruster axis, opposite to the neutralizer position (blue dotted line). (b) Zoom on the slow CEX ions distribution function at the thruster axis: total (solid line), singly charged (dashed line), and doubly charged (dotted line) contributions. All results are instantaneous and not time averaged.

The electron thermodynamics affects both the equilibrium S/C potential and the total current (due to ions and electrons) to the $\mathrm{S} / \mathrm{C}$ walls (including thruster grid, neutralizer and solar arrays), as shown in Fig. 10 (a) and (b). After a short transient, the electric potential of the $\mathrm{S} / \mathrm{C}$ reaches a stationary value, which means that the total current to the $\mathrm{S} / \mathrm{C}$ node ( $I$ in Fig. 4) approaches zero or fluctuates dimly around it. Moreover, the floating potential is quite stable for all $\gamma$ cases (the chosen value for the charging capacity $C$ is $20 \mathrm{nF}$ ).

The larger the polytropic coefficient, the less negative the $\mathrm{S} / \mathrm{C}$ floats, as suggested by the limit to which the electric potential tends when the electron density goes to zero, which is $-T_{e 0} \gamma /(\gamma-1)$, as shown in Ref. [12, 13]. Clearly, since the collected electron current is affected by this limit, the floating potential of the S/C also adapts to it. Moreover, the closer to isothermal the electrons, the larger the collected ion and electron currents, because of the larger electric fields that are capable of deviating a larger fraction of slow ions towards the spacecraft. The transition from the (only) quasineutral solution to a non neutral one is also clear in Fig. 10 (b), where a sudden increase of up to $20 \%$ in the collected ion/electron current is registered, when activating the non-neutral solver (at $t=1 \mathrm{~ms}$ ). Therefore, solving for the finite non-neutral plasma sheaths that surround the $\mathrm{S} / \mathrm{C}$, has a non-negligible influence on the ion flux impinging the spacecraft. 

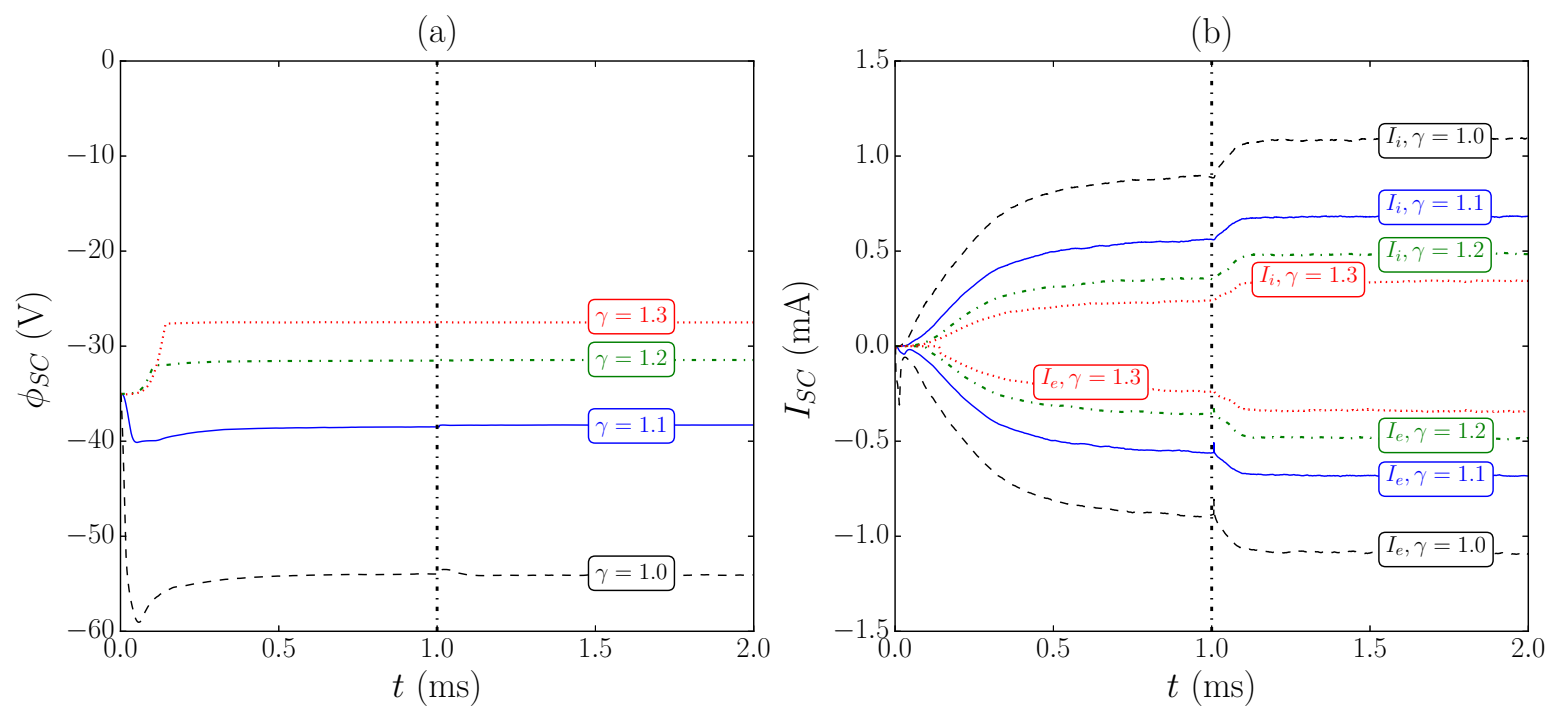

Figure 10: Time averaged evolutions (over 100 steps) of (a) S/C potential and (b) electron and ion current to the $\mathrm{S} / \mathrm{C}$ walls (including thruster grid and case, neutralizer and solar arrays), for the different $\gamma$ cases: isothermal (black dashed line), $\gamma=1.1$ (blue solid line), $\gamma=1.2$ (green dash-dot line), and $\gamma=1.3$ (red dotted line). A vertical dash-dot line indicates when the non-neutral solver is activated $(t=1 \mathrm{~ms})$.

\section{Conclusions}

This paper has presented a highly flexible 3D hybrid model to study the current neutralization and the interaction of a plasma plume with any nearby object, featuring:

- A weakly-collisional electron model admitting a polytropic fitting for the electron pressure tensor, which permits obtaining both the corrected electric potential (due to collisional effects) and the electric current density in the plume.

- An adaptive algorithm that splits the simulation domain into quasineutral and non-neutral regions and handles a smooth transition between them. While in the former the fluid closure relies on the sole electron momentum balance equation, in the latter, this is coupled with Poisson equation.

- A correct transition between quasineutral material boundaries, where Bohm condition is applied, and non-neutral spatially-resolved plasma sheaths.

- Use of both volumetric and surface weighting algorithms for respectively the inner mesh nodes and the boundary faces.

- Treatment of CEX collisions with a DSMC approach, which permits tracking also the fast neutrals.

- Macro-particle population control, based on a generation macro-particle weight, to limit the statistical noise of the PIC algorithms.

A benchmark simulation has then permitted to validate the code, showing that results for the CEX ion density are definitely consistent with available data in the literature. Moreover, it has highlighted that both the electron thermodynamics and the non-neutral plasma regions 
play a non-negligible role in determining the ion flux impinging the spacecraft. Regarding the former, the closer to isothermal are the electrons, the higher this flux and the average wallimpact energy, and the lower the spacecraft floating potential. A significant backscattered CEX ions flux increase is systematically found when switching from a quasineutral solution to a non-neutral one.

Future developments of the model and code should finally tackle the following aspects:

- New heavy particles collisions, such as the momentum exchange collisions between ions and neutrals (MEX).

- A new wall type characterized by sputtering of both neutral and charged material particles.

- A more effective population control to deal with plume expansion effects. This should feature, on top of the already existing control, a particle renormalization algorithm.

- The extension of the electron model to magnetized plasma plumes, in order to study the distorsion effects caused by any applied external magnetic field (such as the geomagnetic field) on the plume expansion.

\section{Acknowledgments}

The research leading to the results of this paper was initiated within the LEOSWEEP project ("Improving Low Earth Orbit Security With Enhanced Electric Propulsion"), funded by the European Union Seventh Framework Programme (FP7/2007-2013) under grant agreement N.607457. Additional funding to complete it has been received by Spain's R\&D National Plan, under grant ESP2016-75887.

\section{Appendix A: models for collisions}

\section{CEX cross section}

The CEX cross section for both types of CEX reactions (singly and doubly charged ions with neutrals) is provided, for Xenon gas, by Mill's model [15]:

$$
\sigma\left(v_{r}\right)=C_{1}-C_{2} \cdot \log _{10}\left(\frac{1 / 2 m v_{r}^{2}}{1 \mathrm{eV}}\right)
$$

where the argument of the logarithm represents the relative kinetic energy of the impacting elementary particles, expressed in $\mathrm{eV}$. The constants depend on the type of reaction:

- $\mathrm{Xe}^{+}($fast $)+\mathrm{Xe}($ slow $) \rightarrow \mathrm{Xe}^{+}($slow $)+\mathrm{Xe}($ fast $) \quad: C_{1}=87.3 \AA^{2}, C_{2}=8.9 \AA^{2}$

- $\mathrm{Xe}^{++}($fast $)+\mathrm{Xe}($ slow $) \rightarrow \mathrm{Xe}^{++}($slow $)+\mathrm{Xe}($ fast $): C_{1}=45.7 \AA^{2}, C_{2}=8.9 \AA^{2}$

\section{Electron collision frequency}

In order to compute the electron momentum transfer collision frequency with the $s^{\text {th }}$ particle population $v_{e s}$, only elastic collisions are taken into account. The electron-ion 
collision frequencies are then obtained as [73]:

$$
v_{e s}=\frac{2^{1 / 2} n_{s} Z_{s}^{2} e^{4} \ln \Lambda}{12 \pi^{3 / 2} \epsilon_{0}^{2} m_{e}^{1 / 2} T_{e}^{3 / 2}}
$$

where $n_{s}$ is $s^{\text {th }}$ population number density, and the parameter $\ln \Lambda \approx 10$. For the neutrals, on the other hand, the collision frequency is computed by integrating the elastic collision cross section $\sigma_{e s}\left(v_{e}\right)$ over a Maxwellian electron distribution function [74, 75]:

$$
v_{e s}=n_{s} \sqrt{\frac{2 T_{e}}{9 \pi m_{e}}} \int_{0}^{\infty}(x)^{5} \sigma_{e s}(x) \exp \left(-\frac{x^{2}}{2}\right) d x
$$

where $x=v_{e} / c_{e}$ represents the normalized electron velocity with respect to the electron thermal velocity $c_{e}=\sqrt{T_{e} / m_{e}}$, and $\sigma_{e s}(x)$ depends on the $s^{\text {th }}$ population atomic type, and follows the model of Ref. [76] (for xenon). It is noticed, that Eqs. 13, 38 and 39 are only valid if the electron thermal velocity $c_{e}$ is much larger than the fluid velocities of both the heavy particle population $u_{s}$ and of the electrons $u_{e}$.

\section{Appendix B: solving the non-linear Poisson equation}

Since the electron density is an explicit function $n_{e}=n_{e}\left(\phi, H_{e}\right)$, of both the known total enthalpy and unknown potential, Eq. 14 becomes a non-linear differential equation in $\phi$. In numerical form, if $\phi_{l}$ is the unknown potential at $l^{\text {th }}$ mesh node, then the $l^{\text {th }}$ non-linear system equation can be written as:

$$
f_{l}=\sum_{m \in \text { mesh }} A_{l m} \phi_{m}+\frac{e}{\epsilon_{0}}\left(n_{e, l}^{*}-n_{e, l}\left(\phi_{l}, H_{e, l}\right)\right)=0
$$

where the summation extends to all mesh nodes, $n_{e, l}^{*}$ is the known quasi-neutral electron density at node $l$, and $A_{l m}$ is the $(l, m)$ element of the sparse coefficients matrix.

Eq. 40 can be solved iteratively with a Newton-Raphson method, by linearizing it around the current solution for the electric potential $\phi^{(k)}$, with $k$ representing now the iteration step. The initial estimate $\phi^{(0)}$ is given by either the quasineutral electric potential $\phi^{*}$ (at the very first simulation step), or by the previous time step solution (at other simulation steps). The linearization proceeds by evaluating the Jacobian matrix of the non-linear system, at the iteration step $k$, as:

$$
\left.J_{l m}^{(k)}=\frac{\partial f_{l}}{\partial \phi_{m}}\right]_{\phi=\phi^{(k)}}
$$

and then obtaining the electric potential correction $\Delta \phi^{(k)}$, by solving the linearized system:

$$
\sum_{m \in \text { mesh }} J_{l m}^{(k)} \Delta \phi_{m}^{(k)}=-f_{l}^{(k)}
$$

The new electric potential, for the next iteration step $k+1$, is updated as $\phi_{l}^{(k+1)}=\phi_{l}^{(k)}+\Delta \phi_{l}^{(k)}$ and used to update the Jacobian matrix. This iterative scheme is repeated until a convergence criterion is met, i.e. until the maximum absolute value of the non linear function $f$ is below a user's defined tolerance. 


\section{References}

[1] D.M. Goebel and I. Katz. Fundamentals of Electric Propulsion: Ion and Hall Thrusters. JPL, 2008.

[2] E. Ahedo. Plasmas for space propulsion. Plasma Physics and Controlled Fusion, 53(12):124037, 2011.

[3] H.B. Garrett. The charging of spacecraft surfaces. Reviews of Geophysics, 19(4):577-616, 1981.

[4] I.D. Boyd and A. Ketsdever. Interactions between spacecraft and thruster plumes. Journal of Spacecraft and Rockets, 38(3):380-380, 2001.

[5] C. Bombardelli and J. Peláez. Ion beam shepherd for contactless space debris removal. Journal of Guidance, Control, and Dynamics, 34(3):916-920, May 2011.

[6] C. Bombardelli, H. Urrutxua, M. Merino, E. Ahedo, and J. Peláez. Relative dynamics and control of an ion beam shepherd satellite. In James V. McAdams, David P. McKinley, Matthew M. Berry, and Keith L. Jenkins, editors, Spaceflight mechanics 2012, volume 143 of Advances in the Astronautical Sciences, pages 2145-2158. Univelt, 2012.

[7] C. Bombardelli, H. Urrutxua, M. Merino, E. Ahedo, and J. Peláez. The ion beam shepherd: A new concept for asteroid deflection. AA, 90(1):98 - 102, 2013.

[8] M. Merino, E. Ahedo, C. Bombardelli, H. Urrutxua, and J. Peláez. Ion beam shepherd satellite for space debris removal. In Luigi T.DeLuca, Christophe B., Oskar J.H., and Sergey M.F., editors, Progress in Propulsion Physics, volume IV of EUCASS Advances in Aerospace Sciences, chapter 8, pages 789-802. Torus Press, 2013.

[9] M.A. Lieberman and A.J. Lichtenberg. Principles of plasma discharges and materials processing. John Wiley \& Sons, 2005.

[10] K. Wilhelm, L. Abbo, F. Auchère, N. Barbey, L. Feng, A.H. Gabriel, S. Giordano, S. Imada, A. Llebaria, W.H. Matthaeus, et al. Morphology, dynamics and plasma parameters of plumes and inter-plume regions in solar coronal holes. The Astronomy and Astrophysics Review, 19(1):35, 2011.

[11] D. Mascali, S. Tudisco, A. Bonanno, N. Gambino, S. Ivanovski, A. Anzalone, S. Gammino, R. Miracoli, and F. Musumeci. Colliding laser-produced plasmas: a new tool for nuclear astrophysics studies. Radiation Effects $\mathcal{F}$ Defects in Solids: Incorporating Plasma Science E Plasma Technology, 165(610):730-736, 2010.

[12] F. Cichocki, M. Merino, and E. Ahedo. Modeling and simulation of EP plasma plume expansion into vacuum. In 50th AIAA/ASME/SAE/ASEE Joint Propulsion Conference, Cleveland, OH, AIAA 20143828, 2014.

[13] M. Merino, F. Cichocki, and E. Ahedo. Collisionless plasma thruster plume expansion model. Plasma Sources Science and Technology, 24(3):035006, 2015.

[14] D. Rapp and W.E. Francis. Charge exchange between gaseous ions and atoms. Journal of Chemical Physics, 37(11):2631-2645, 1962.

[15] J.S. Miller. Xenon charge exchange cross sections for electrostatic thruster models. Journal of applied physics, 91(3):984-991, 2002.

[16] D.E. Parks and I. Katz. A preliminary model of ion beam neutralization. In 14th International Electric Propulsion Conference, Fairview Park, OH, 1979. Electric Rocket Propulsion Society.

[17] J. Ashkenazy and A. Fruchtman. Plasma plume far field analysis. In 27th International Electric Propulsion Conference, Fairview Park, OH, 2001. Electric Rocket Propulsion Society.

[18] A.G. Korsun and E.M. Tverdokhlebova. The characteristics of the EP exhaust plume in space. In 33rd AIAA/ASME/SAE/ASEE Joint Propulsion Conference $\mathcal{E}$ Exhibit, Washington DC, 1997. AIAA.

[19] K. Dannenmayer, S. Mazouffre, E. Ahedo, and M. Merino. Hall effect thruster plasma plume characterization with probe measurements and self-similar fluid models. In 48th AIAA/ASME/SAE/ASEE Joint Propulsion Conference $\mathcal{E}$ Exhibit, number AIAA 2012-4117, Atlanta, Georgia, 2012. AIAA.

[20] F. Cichocki, M. Merino, E. Ahedo, D. Feili, and M. Ruiz. Electric propulsion subsystem optimization for "Ion Beam Shepherd" missions. In 34th International Electric Propulsion Conference and 6th Nanosatellite Symposium, Hyogo-Kobe, Japan, number IEPC-2015-420, 2015.

[21] A.L. Ortega, I. Katz, I.G. Mikellides, and D.M. Goebel. Self-consistent model of a high-power hall thruster plume. IEEE Transactions on Plasma Science, 43(9):2875-2886, 2015. 
[22] M. Martínez-Sánchez, J. Navarro-Cavallé, and E. Ahedo. Electron cooling and finite potential drop in a magnetized plasma expansion. Physics of Plasmas, 22:053501, 2015.

[23] Y. Hu and J. Wang. Fully kinetic simulations of collisionless, mesothermal plasma expansion. In 13th Spacecraft Charging Technology Conf., 2014.

[24] J. Wang, D. Han, and Y. Hu. Kinetic simulations of plasma plume potential in a vacuum chamber. IEEE Transactions on Plasma Science, 43(9):3047-3053, 2015.

[25] A.G. Korsun, E.M. Tverdokhlebova, and F.F. Gabdullin. Mathematical model of hypersonic plasma flows expanding in vacuum. Computer physics communications, 164(1-3):434-441, 2004.

[26] D.Y. Oh, D.E. Hasting, C.M. Marrese, J.M. Haas, and A.D. Gallimore. Modeling of stationary plasma thruster-100 thruster plumes and implications for satellite design. Journal of Propulsion and Power, 15(2):345-357, 1999.

[27] J. Forest, L. Eliasson, and A. Hilgers. A new spacecraft plasma simulation software, picup3d/spis. In Spacecraft Charging Technology, volume 476, page 515, 2001.

[28] M. Masselin. Development of a hybrid pic code for the simulation of plasma spacecraft interactions, 2012.

[29] M. Wartelski, C. Theroude, C. Ardura, and E. Gengembre. „self-consistent simulations of interactions between spacecraft and plumes of electric thrusters". Technical report, IEPC-2013, 2013.

[30] L. Garrigues, J. Bareilles, J.P. Boeuf, and I.D. Boyd. Modeling of the plasma jet of a stationary plasma thruster. Journal of applied physics, 91(12):9521-9528, 2002.

[31] M. Celik, M. Santi, S. Cheng, M. Martínez-Sánchez, and J. Peraire. Hybrid-pic simulation of a Hall thruster plume on an unstructured grid with dsmc collisions. In 28th International Electric Propulsion Conference, Toulouse, France, IEPC-03-134, 2003.

[32] S. Cheng, M. Santi, M. Celik, M. Martinez-Sanchez, and J. Peraire. Hybrid pic-dsmc simulation of a hall thruster plume on unstructured grids. Computer physics communications, 164(1):73-79, 2004.

[33] L. Brieda, J. Pierru, R. Kafafy, and J. Wang. Development of the draco code for modeling electric propulsion plume interactions. In 40th AIAA/ASME/SAE/ASEE Joint Propulsion Conference and Exhibit, page 3633, 2004.

[34] F. Taccogna, D. Pagano, F. Scortecci, and A. Garulli. Three-dimensional plume simulation of multichannel thruster configuration. Plasma Sources Science and Technology, 23(6):065034, 2014.

[35] B. Korkut and D.A. Levin. Three dimensional coupled pic and dsmc simulations of ion thruster plumes with sugar. In 50th AIAA/ASME/SAE/ASEE Joint Propulsion Conference, page 3447, 2014.

[36] B. Korkut, Z. Li, and D.A. Levin. 3-d simulation of ion thruster plumes using octree adaptive mesh refinement. IEEE Transactions on Plasma Science, 43(5):1706-1721, 2015.

[37] O. Kalentev, K. Matyash, J. Duras, K.F. Lüskow, R. Schneider, N. Koch, and M. Schirra. Electrostatic ion thrusters-towards predictive modeling. Contributions to Plasma Physics, 54(2):235-248, 2014.

[38] D.L. Kahnfeld. Hybrid Plume Modeling. Master's thesis, Mathematisch-Naturwissenschaftliche Fakultät Ernst-Moritz-Arndt-Universität Greifswald, Greifswald, Germany, 2015.

[39] C. Cai. Numerical studies on plasma plume flows from a cluster of electric propulsion devices. Aerospace Science and Technology, 41:134-143, 2015.

[40] S.J. Araki, R.S. Martin, D. Bilyeu, and J.W. Koo. Sm/murf: Current capabilities and verification as a replacement of afrl plume simulation tool coliseum. In 52nd AIAA/SAE/ASEE Joint Propulsion Conference, page 4939, 2016.

[41] I. Boyd and J. Yim. Hall thruster plume simulation using a detailed hybrid model. In 40th AIAA/ASME/SAE/ASEE Joint Propulsion Conference and Exhibit, page 3952, 2004.

[42] F. Cichocki, A. Domínguez, M. Merino, and E. Ahedo. A 3D hybrid code to study electric thruster plumes. In Space Propulsion Conference, 2016.

[43] M.M. Santi. Hall thruster plume simulation using a hybrid-PIC algorithm. PhD thesis, Massachusetts Institute of Technology, 2003.

[44] F.I. Parra, E. Ahedo, J.M. Fife, and M. Martínez-Sánchez. A two-dimensional hybrid model of the hall thruster discharge. Journal of Applied Physics, 100(2):023304, 2006.

[45] F. Parra, D. Escobar, and E. Ahedo. Improvements on particle accuracy in a hall thruster hybrid code. In 42th Joint Propulsion Conference, Sacramento, CA, 2006. 
[46] R. Santos and E. Ahedo. Accuracy improvements in a hall thruster pic/fluid code. In 45th AIAA/ASME/SAE/ASEE Joint Propulsion Conference E Exhibit, page 4914, 2009.

[47] E. Ahedo, R. Santos, and F. Parra. Fulfilment of the kinetic bohm criterion in a quasineutral particle-in-cell model. Physics of Plasmas, 17(7):073507, 2010.

[48] R. Santos and E. Ahedo. Implementation of the kinetic bohm condition in a Hall thruster hybrid code. In 45th Joint Propulsion Conference, Denver, CO, AIAA 2009-4913, 2009.

[49] G.A. Bird. Molecular gas dynamics and the direct simulation of gas flows. 1994. clarendon, 1994.

[50] D. Tskhakaya, K. Matyash, R. Schneider, and F. Taccogna. The particle-in-cell method. Contributions to Plasma Physics, 47(8-9):563-594, 2007.

[51] R.W. Hockney and J.W. Eastwood. Computer simulation using particles. crc Press, 1988.

[52] C.K. Birdsall and D. Fuss. Clouds-in-clouds, clouds-in-cells physics for many-body plasma simulation. Journal of Computational Physics, 135(2):141-148, 1997.

[53] K. Nanbu and Y. Kitatani. An ion-neutral species collision model for particle simulation of glow discharge. Journal of Physics D: Applied Physics, 28(2):324, 1995.

[54] Y. Azziz, M. Martinez-Sanchez, and J. Szabo. Determination of in-orbit plume characteristics from laboratory measurements. In 42nd AIAA/ASME/SAE/ASEE Joint Propulsion Conference E Exhibit, page 4484, 2006.

[55] M. Mitchner and C.H. Kruger Jr., editors. Partially ionized gases. Wiley, 1973.

[56] E.W. Bell, N. Djurić, and G.H. Dunn. Electron-impact ionization of $\mathrm{in}^{+}$and $\mathrm{xe}^{+}$. Physical Review A, 48(6):4286, 1993.

[57] E.R. Harrison and W.B. Thompson. The low pressure plane symmetric discharge. Proceedings of the Physical Society, 74(2):145, 1959.

[58] Giovanni Lapenta. Particle rezoning for multidimensional kinetic particle-in-cell simulations. Journal of computational physics, 181(1):317-337, 2002.

[59] R. S. Martin and J.L. Cambier. Octree particle management for dsme and pic simulations. Journal of Computational Physics, 327:943-966, 2016.

[60] J. Navarro-Cavallé, M. Martínez-Sánchez, and E. Ahedo. Collisionless electron cooling in a magnetic nozzle. In 50th AIAA/ASME/SAE/ASEE Joint Propulsion Conference, Cleveland, Ohio, 2014. AIAA.

[61] M. Merino, P. Fajardo, and E. Ahedo. Collisionless electron cooling in unmagnetized plasma thruster plumes. In 52nd AIAA/SAE/ASEE Joint Propulsion Conference, page 5037, 2016.

[62] Y. Hu and J. Wang. Electron properties in collisionless, mesothermal plasma expansion: Fully kinetic simulations. IEEE Trans. Plasma Science, 2015.

[63] J. Navarro-Cavallé, S. Correyero, and E. Ahedo. Collisionless electron cooling on magnetized plasma expansions: advances on modelling. In 34th International Electric Propulsion Conference, 2015.

[64] J. Polk, R. Kakuda, J. Anderson, J. Brophy, V. Rawlin, M. Patterson, J. Sovey, and J. Hamley. Validation of the nstar ion propulsion system on the deep space one mission-overview and initial results. In 35th Joint Propulsion Conference and Exhibit, page 2274, 1999.

[65] J.R. Brophy. Nasa's deep space 1 ion engine (plenary). Review of Scientific Instruments, 73(2):1071-1078, 2002.

[66] J.E. Foster, G.J. Williams, and M.J. Patterson. Characterization of an ion thruster neutralizer. Journal of propulsion and power, 23(4):828-835, 2007.

[67] J.R. Brophy, C.E. Garner, and S.C. Mikes. Dawn ion propulsion system: Initial checkout after launch. Journal of Propulsion and Power, 25(6):1189-1202, 2009.

[68] J. Snyder, D.M. Goebel, R.R. Hofer, J.E. Polk, N.C. Wallace, and H. Simpson. Performance evaluation of the t6 ion engine. Journal of Propulsion and Power, 28(2):371-379, 2012.

[69] R.I.S. Roy, D.E. Hastings, and N.A. Gastonis. Ion-thruster plume modeling for backflow contamination. Journal of Spacecraft and Rockets, 33(4):525-534, 1996.

[70] R.I.S. Roy, D.E. Hastings, and N.A. Gastonis. Numerical study of spacecraft contamination and interactions by ion-thruster effluents. Journal of Spacecraft and Rockets, 33(4):535-542, 1996.

[71] J. Wang, D. Brinza, and M. Young. Three-dimensional particle simulation modeling of ion propulsion plasma environment for deep space one. \{Journal of Spacecraft and Rockets\}.volume, (3), 2001. 
[72] F. Taccogna and G. Dilecce. Non-equilibrium in low-temperature plasmas. The European Physical Journal $D, 70(11): 251,2016$.

[73] R.J. Goldston and P.H. Rutherford. Introduction to Plasma Physics. Institute of Physics Publishing, Bristol, 1995.

[74] P.M. Bellan. Fundamentals of Plasma Physics. 2006. Cambridge University Press.

[75] J.A. Bittencourt. Fundamentals of plasma physics. Springer Science \& Business Media, 2013.

[76] M. Hayashi. Bibliography of electron and photon cross sections with atoms and molecules published in the 20th century. argon. Technical report, National Inst. for Fusion Science, 2003. 